\title{
TANGENT CONES TO DISCRIMINANT LOCI FOR FAMILIES OF HYPERSURFACES
}

\author{
ROY SMITH AND ROBERT VARLEY
}

\begin{abstract}
A deformation of a variety with (nonisolated) hypersurface singularities, such as a projective hypersurface or a theta divisor of an abelian variety, determines a rational map of the singular locus to projective space and the resulting projective geometry of the singular locus describes how the singularities propagate in the deformation. The basic principle is that the projective model of the singular locus is dual to the tangent cone to the discriminant of the deformation. A detailed study of the method, which emerged from interpreting Andreotti-Mayer's work on theta divisors in terms of Schlessinger's deformation theory of singularities, is given along with examples, applications, and multiplicity formulas.
\end{abstract}

Introduction. This paper is about the geometry associated to deformations of nonisolated hypersurface singularities. The basic principle is that a deformation of a "hypersurface" determines a projective model of the singular locus whose dual variety is the projectivized tangent cone to the discriminant. This includes an infinitesimal form of Bertini's theorem for a linear system of divisors and a converse to Andreotti-Mayer's condition for double points of theta divisors.

Now we describe the main results more precisely, working in the category of complex analytic spaces. Let $X$ be a space which can locally be written as a hypersurface of a nonsingular space and let $\mathbf{X} \stackrel{\pi}{\rightarrow} S$ be a deformation of $X=$ $\pi^{-1}(0)$. Then $\mathbf{T}_{X}^{1}$, the sheaf of first order deformations of $X$, is a line bundle on sg. $X$, the singular locus of $X$, and $\pi$ determines a Kodaira-Spencer linear map $T_{0}(S) \rightarrow H^{0}\left(\mathbf{T}_{X}^{1}\right)$, i.e. a linear system on the singular locus. If $\mathbf{X}$ and $S$ are nonsingular this linear system has no base locus and the corresponding morphism $\phi:$ sg. $X \rightarrow \mathbf{P} T_{0}^{*}(S)$ satisfies $\phi(p)=\left[\pi_{*, p}\left(T_{p} \mathbf{X}\right)\right]$ for $p \in \operatorname{sg} . X$, hence is called the Gauss map of $\pi$. For a projective hypersurface of degree $d, \phi$ is the $d$-fold Veronese map restricted to the singular locus, and for the theta divisor of an abelian variety, $\phi$ is the rational map assigning to a double point its quadric tangent cone.

Assuming the restriction of $\pi$ to the critical locus $\mathbf{C} \subset \mathbf{X}$ is proper, consider the discriminant locus $D=\pi(\mathbf{C})$ with its reduced analytic structure. In addition to the above, suppose finally that $\mathbf{C}$ is nonsingular of the expected codimension in $\mathbf{X}$.

DUALITY THEOREM. There is a finite collection of subvarieties $\left\{Z_{\alpha}\right\}$ of sg. $X$, which is intrinsic to the analytic space sg. $X$ and includes the irreducible components,

Received by the editors March 31, 1987. The contents of this paper were presented October 18, 1986 by the first author in the special session on singularities and algebraic geometry at the Charlotte, North Carolina, meeting of the AMS.

1980 Mathematics Subject Classification (1985 Revision). Primary 14D15, 14N05; Secondary 32G11, 53C57. 
such that, as sets,

$$
\mathrm{P} C_{0}(D)=\bigcup_{\alpha} \phi\left(Z_{\alpha}\right)^{*}
$$

i.e. the projectivized tangent cone at 0 to the discriminant $D \subset S$ is set-theoretically the union of the dual varieties of $\phi\left(Z_{\alpha}\right) \subset \mathbb{P} T_{0}^{*}(S)$.

For the versal deformation of a (semilocal) hypersurface with isolated singularities this reduces to the well-known result [T1] that, as set, the tangent cone to the discriminant is a union of distinct hyperplanes, one for each singular point. Further, each hyperplane is known to occur in the scheme-theoretic tangent cone with multiplicity equal to the Milnor number of the corresponding isolated hypersurface singularity; a multiplicity formula can be given in the general case also.

When sg. $X$ is smooth (the case of an "ordinary double locus" of $X$ ), the proofs of the duality theorem and multiplicity formula can be based directly on the Fréchet derivative of the map $\mathbf{C} \rightarrow D$ along the fibre sg. $X$ over 0 and we have included a discussion of this method. Applications of the duality theorem to projective hypersurfaces and theta divisors are given in the last section.

In the rest of the introduction, we would like to indicate the method of proof of the duality theorem and the relation to other work. As we were working out the general formulation of the principle for tangent cones to discriminant loci we learned of the local projective duality theorem of Lê and Teissier, announced in $[\mathbf{T 4}, \S 1]$. Their result expresses, for any reduced, equidimensional subspace of a smooth analytic space, a duality between the projectivized tangent cone at a point and the fibre of the conormal variety over the point. Assuming this, our result can be viewed as describing the conormal variety of the discriminant locus for deformations of hypersurface singularities. Namely, the conormal variety is the image of the critical locus under the Gauss map. This was known for the versal deformation of isolated hypersurface singularities [T3, 5.5.1; L, 4.C] and similar constructions are used in symplectic geometry [Wei, pp. 25-26; A2, p. 31]. Now our proof of the duality theorem runs as follows: since C maps to a "Legendre" subvariety [A1, Appendix $4 \mathrm{~K}$ ] of $\mathbb{P} T^{*}(S)$, the same should be true on exceptional divisors after blowing up over $0 \in S$. More precisely, let $\mathscr{E}$ be the exceptional divisor in the blowup of $\mathbf{C}$ along sg. $X$ and note that the exceptional divisor in the blowup of $\mathbb{P} T^{*}(S)$ along $\mathbb{P} T_{0}^{*}(S)$ is $\mathbb{P} T_{0}^{*}(S) \times \mathbb{P} T_{0}(S)$. We relate the natural contact structures on $\mathrm{P}^{*}(S)$ and the incidence variety $I \subset \mathrm{P} T_{0}^{*}(S) \times \mathbb{P} T_{0}(S)$. Then we conclude, by a specialization argument analogous to Kleiman's [Kl1, 3.8], the image of $\mathscr{E}_{\text {red. }}$ is Legendre in $I$, i.e. a union of dual correspondences. The method is similar to the second proof given in [L-T, Théorème 2.1.1 and Corollaire 2.1.3] and to the proof (C. Sabbah) indicated in [K12, p. 220].

Recently, it was suggested that the duality theorem we have given seems to be a special case of theorems (cf. Sabbah $[\mathbf{S}]$ ) about direct images of Lagrangian cycles. It is certainly interesting to relate deformation theory of singularities to constructions in the theory of $\mathbf{D}$-modules and we hope to discuss this, together with further applications of our methods, in the future.

A CKnowledgments. We are grateful to H. Clemens, A. Collino, R. Donagi, H. Hironaka, G. Kempf, G. Kennedy, S. Kleiman, A. Landman, Lê D. T., C. McCrory, T. Shifrin, M. Spivakovsky, and B. Teissier for discussion about this work and 
particularly to M. Schlessinger for encouragement. Both authors were partially supported by NSF Grants DMS-83-17078 and DMS-8603281.

1. The basic setup. Let $\pi: \mathrm{X} \rightarrow S$ be a family of varieties with only hypersurface singularities; i.e. $\mathbf{X}$ and $S$ are analytic spaces, $\pi$ is an analytic map, and for all $x \in \mathbf{X}$, if $s=\pi(x) \in S$, there are open sets $U$ and $V$, with $s \in V \subseteq S$, and $x \in U \subseteq \pi^{-1}(V) \subseteq \mathbf{X}$, such that, on $U, \pi$ is isomorphic to the projection $V \times \mathbb{C}^{n} \rightarrow V$ restricted to the zero locus of one analytic equation $F$ in an open subset of $V \times \mathbb{C}^{n}$ :

$$
\begin{aligned}
& U \cong\{F=0\} \subseteq V \times \mathbb{C}^{n} \\
& \pi \downarrow \\
& V
\end{aligned}
$$

The locus " $F=0$ " in an open subset $\mathbf{W} \subset V \times \mathbb{C}^{n}$ means the analytic space which is the zero set of $F$ in $\mathbf{W}$ endowed with the structure that $\mathbf{O}_{\mathbf{W}} /(F)$. We require for each $t_{0} \in V$, that $F\left(t_{0} ; x_{1}, \ldots, x_{n}\right)$ not be the zero germ at any point of $\mathbf{W} \cap\left(\left\{t_{0}\right\} \times \mathbb{C}^{n}\right)$. In particular, $\pi$ is flat [Mu1, Example P, pp. 431-432; D, Chapter I, pp. 47-62] and so, by definition, is a deformation of each of its fibers. We will call a single fiber $\pi^{-1}(s)=X_{s}$ of $\pi$ (locally) a hypersurface. Conversely, if $\pi: \mathbf{X} \rightarrow(S, 0)$ is a (small) deformation of a "hypersurface", then it is a family of varieties with only hypersurface singularities; cf. [Schl2, 1.1].

We define the critical locus $\mathbf{C} \subseteq \mathbf{X}$ of the family by the vanishing of the "vertical" partials of the local equation; i.e., after embedding $U$ in $V \times \mathbb{C}^{n}$ as above, $\mathbf{C} \cap U$ has equations $\left\{F=\partial F / \partial x_{1}=\cdots=\partial F / \partial x_{n}=0\right\}$. It can be immediately checked that $\mathbf{C}$ is a well-defined analytic subspace of $\mathbf{X}$ which can also be given an intrinsic definition using a Fitting ideal of $\Omega_{\mathbf{X} / S}^{1}[\mathbf{T 3}, \S 2.5]$. We define the discriminant $D \subseteq S$, as a set, to be the image $\pi(\mathbf{C})$ of the critical locus. We denote the restriction of $\pi$ to $\mathbf{C}$ by $\eta$ : $\mathbf{C} \rightarrow S$. Assuming $\eta$ is proper, $D$ is an analytic subspace of $S$ [Wh, Chapter 5, §4] which we consider with its reduced induced structure.

For each $s \in S$ we denote the scheme-theoretic fiber of $\eta$ by $\eta^{-1}(s)=\mathrm{sg} . X_{s}$, the "singular locus" or "singular scheme" of the hypersurface $X_{s}$. These constructions are displayed in the following diagram:

$$
\begin{array}{ccccccc}
X_{s} & \subseteq & \mathbf{X} & \supseteq & \mathbf{C} & \supseteq & \text { sg. } X_{s}=X_{s} \cap \mathbf{C} \\
\downarrow & & \downarrow & & \downarrow & & \downarrow \\
s & \in & S & \supseteq & D & \ni & s
\end{array}
$$

REMARK (1.1). If $\eta: \mathbf{C} \rightarrow D$ is a finite map, then $D$ can be given a natural structure of (possibly nonreduced) analytic space [T3, §2.6], but since we are particularly interested in the case of nonisolated singularities, we will not assume that $\mathbf{C}$ is finite over $D$, but only proper. Thus the singular schemes sg. $X_{s}$ that we consider will be complete, but not necessarily finite. Generally, we expect the discriminant to have pure codimension one in $S$, and this is true provided, say, $\mathrm{X}$ and $S$ are smooth, $D \neq \varnothing$, and the generic hypersurface over $D$ has isolated singularities. A standard exception is provided [Mu4] by a nonsingular subvariety $V \subseteq \mathbb{P}^{N}$, in projective space, whose dual variety is not a hypersurface. In that case the family of hyperplane sections of $V$ has discriminant locus $V^{*} \subseteq\left(\mathbf{P}^{N}\right)^{*}$ of codimension higher than one.

Our main object in this paper is the following: given a base point $0 \in D$, to describe geometrically the projectivized tangent cone $\mathrm{P} C_{0}(D)$ to $D$ at 0 , and relate 
the description to the deformations of the singular points of $X_{0}$. In particular we will try to answer these questions:

1. Along which directions in $\mathbb{P} C_{0}(D)$ do all singular points of $X_{0}$ deform?

2. Given $p \in \operatorname{sg} . X_{0}$, along which directions does $p$ deform?

3. What are the smoothing directions for $X_{0}$ ?

There are two classical examples of particular interest to us.

EXAMPLE (i). The universal projective hypersurface: let $\mathbb{P}^{N}$ be projective $n$-space, let $\mathbb{P}(n, d)=\mathbb{P}\left(H^{0}\left(\mathbb{P}^{n} ; \mathbf{O}(d)\right)\right)$ be the projective space parametrizing (scheme-theoretic) hypersurfaces of degree $d$ in $\mathbb{P}^{n}$, and let

$$
F(x, a)=\sum_{|I|=d} a_{I} x^{I}
$$

be the general homogeneous polynomial of degree $d$ on $\mathbb{P}^{n}$. Then

$$
\begin{aligned}
& \mathbf{X}=\{F=0\} \subseteq \mathbb{P}^{n} \times \mathbb{P}(n, d) \\
& \pi \downarrow \\
& \mathbb{P}(n, d)
\end{aligned}
$$

is the corresponding universal family. In this situation the discriminant locus $D \subseteq$ $\mathbb{P}(n, d)$ is defined by the classical discriminant of a polynomial in several variables.

EXAMPLE (ii). The universal theta divisor over the Siegel upper half-space $\mathscr{H}_{g}$. To be precise, if

$$
\mathscr{H}_{g}=\left\{\Omega \in \mathbb{G}^{g \times g}:{ }^{t} \Omega=\Omega \text { and } \operatorname{Im} \Omega \gg 0\right\}
$$

is the Siegel upper half-space, let

$$
\theta(z, \Omega)=\sum_{n \in \mathbb{Z}^{g}} \exp \left[i \pi\left({ }^{t} n \Omega n+2^{t} n z\right)\right]
$$

be the Riemann theta function, and let

$$
\begin{aligned}
& \boldsymbol{\Theta} \\
& \downarrow \\
& \mathscr{\mathscr { H }}_{g}
\end{aligned}
$$

be the corresponding family of theta divisors. In this example, the discriminant locus was introduced, as " $\mathbf{N}_{0}$ ", in the very influential paper $[\mathbf{A}-\mathbf{M}]$, and has subsequently played an important role in the study of the moduli spaces of principally polarized abelian varieties.

2. Deformation theory. Next we introduce the machinery [Schl1] for measuring to first order how the individual fibers of a family vary as we deform a hypersurface $X$. Let $\pi: \mathbf{X} \rightarrow S$ be a family as in $\S 1$, and $X=X_{s}$ a particular fiber. Then define $\mathbf{T}_{X}^{1}=$ the sheaf of first order deformations of $X$, to be the sheaf associated to the presheaf:

$(U \subseteq X) \mapsto\left\{\right.$ the vector space (and $\Gamma\left(\mathbf{O}_{U}\right)$-module)

of isomorphism classes of first order deformations of $U$ \}

[Schl1, 2.2.3, pp. 26-33; Schl2, 1.1-1.2; Schu, §§1-2]. This is a sheaf of $\mathbf{O}_{X^{-}}$ modules and we intend particularly to exploit the module structure of the sheaf $\mathbf{T}_{X}^{1}$.

Next we give the "global" calculation of $\mathbf{T}_{X}^{1}$ in terms of the normal bundle to the hypersurface in a smooth ambient space: 
LEMMA (2.1). If $X^{n-1} \subseteq M^{n}$ is a hypersurface in a nonsingular space, then

$$
\mathbf{T}_{X}^{1} \cong\left(\mathbf{N}_{X / M}\right)_{\mid \mathbf{s g} .(X)}
$$

is the restriction, to the singular scheme of $X$, of the normal line bundle to $X$ in $M$.

Proof. We start from the fundamental exact sequence for $\mathbf{T}^{1}$ [Schl2, p. 149; Schu, p. 268]:

$$
0 \rightarrow \mathbf{T}_{X}^{0} \rightarrow\left(\mathbf{T}_{M}^{0}\right)_{\mid X} \rightarrow \mathbf{N}_{X / M} \rightarrow \mathbf{T}_{X}^{1} \rightarrow 0 .
$$

Claim. $\mathbf{T}_{X}^{1}$ has the presentation

$$
0 \rightarrow \mathbf{J} \cdot \mathbf{N}_{X / M} \rightarrow \mathbf{N}_{X / M} \rightarrow \mathbf{T}_{X}^{1} \rightarrow 0,
$$

where $\mathbf{J} \subseteq \mathbf{O}_{X}$ is the jacobian ideal defining the subscheme sg. $X \subseteq X$.

PROOF OF CLAIM. Recall that $\mathrm{N}_{X / M} \cong \operatorname{Hom}_{\mathbf{O}_{X}}\left(I_{X} / I_{X}^{2}, \mathbf{O}_{X}\right)$ where $I_{X}$ is the locally principal ideal sheaf of $X$ in $M$, and the map in (*) is given by

$$
\left.\left(\mathbf{T}_{M}^{0}\right)\right|_{X} \rightarrow \mathbf{N}_{X / M}, \quad D \mapsto(f \mapsto D(f))
$$

where $D$ is a derivation and $f$ is a local equation for $X$ in $M$. Consequently, the image of this map is generated by the partials of $f$ multiplied by $(f \mapsto 1)$, a local basis element of $\mathbf{N}_{X / M}$. Thus the image of this map, which by exactness of $(*)$ is the kernel of $\mathbf{N}_{X / M} \rightarrow \mathbf{T}_{X}^{1}$, equals $\left(\mathbf{J} \cdot \mathbf{N}_{X / M}\right)$. That proves the Claim, and since $\mathbf{J}$ defines sg. $X$, we have

$$
\begin{aligned}
& \mathbf{T}_{X}^{1} \cong\left(\mathbf{N}_{X / M}\right) /\left(\mathbf{J} \cdot \mathbf{N}_{N / M}\right) \cong\left(\mathbf{N}_{X / M}\right) \otimes_{\mathbf{O}_{X}}\left(\mathbf{O}_{X / \mathbf{J}}\right)=\left.\left(\mathbf{N}_{X / M}\right)\right|_{\text {sg. } X} \text {. Q.E.D. } \\
& \text { COROLLARY (2.2). } \mathbf{T}_{X}^{1} \text { is a line bundle on } \mathrm{sg} . X .
\end{aligned}
$$

Since $\mathbf{T}_{X}^{1}$ is a line bundle on sg. $X$, it is locally isomorphic to $\mathbf{O}_{\text {sg. } X}$, which has the local expression

$$
\left(\mathbf{O}_{\text {sg. } X}\right)_{\mid U} \cong \mathbf{O}_{U} /\left(\partial f / \partial x_{1}, \ldots, \partial f / \partial x_{n}\right) \cong \mathbf{O}_{W} /\left(f, \partial f / \partial x_{1}, \ldots, \partial f / \partial x_{n}\right),
$$

where $W \subseteq \mathbb{C}^{n}$ is open, and $X$ is isomorphic, in the open subset $U \subseteq X$, to the hypersurface defined by $f$ in $W$ :

$$
U \cong\{f=0\} \subseteq W \subseteq \mathbb{C}^{n}
$$

Since we will make local calculations we first spell out the local isomorphisms explicitly: in terms of the normal bundle expression we have

$$
\begin{aligned}
\left(\mathbf{N}_{X / M}\right)_{\text {|sg. } X} & \rightarrow \mathbf{O}_{W} /\left(f, \partial f / \partial x_{1}, \ldots, \partial f / \partial x_{n}\right) \\
(f & \mapsto g) \mapsto g
\end{aligned}
$$

and in terms of first order deformations we have

$$
\begin{gathered}
\mathbf{O}_{W} /\left(f, \partial f / \partial x_{1}, \ldots, \partial f / \partial x_{n}\right) \rightarrow \mathbf{T}_{X}^{1} \\
g \mapsto(f+g \cdot \varepsilon)
\end{gathered}
$$

More precisely, a first order deformation of $X$ is a commutative diagram

$$
\begin{aligned}
& \mathbf{X} \supseteq X \\
& \pi \downarrow \downarrow \\
& \operatorname{spec}(\mathbb{C}[\varepsilon]) \ni 0
\end{aligned}
$$


where $\pi$ is a flat map; and if

$$
X=\{f=0\} \subseteq W \subseteq \mathbb{C}^{n} \text { where } f, g \in \mathbf{O}_{W},
$$

then $(f+g \cdot \varepsilon)$ in $\mathbf{O}_{W}[\varepsilon]$ defines a family in $W \times \operatorname{spec}(\mathbb{C}[\varepsilon])$ which is a first order deformation:

$$
\begin{aligned}
& \mathbf{X}=\{f+g \cdot \varepsilon=0\} \subseteq W \times \operatorname{spec}(\mathbb{C}[\varepsilon]) \\
& \downarrow \\
& \operatorname{spec}(\mathbb{C}[\varepsilon]) .
\end{aligned}
$$

Then two such deformations are isomorphic if and only if the two $g$ 's differ by an element of the jacobian ideal.

Now that we have exhibited an intrinsic line bundle $\mathbf{T}_{X}^{1}$ on sg. $X$, we will examine the sections of this bundle arising from the first order deformations induced by $\pi: \mathbf{X} \rightarrow S$. These come from the following version of the Kodaira-Spencer map:

LEMMA (2.3). (i) The family $\pi: \mathbf{X} \rightarrow S$ induces, for each point $0 \in S$ and $X=\pi^{-1}(0)$, a linear map

$$
\begin{gathered}
\rho: T_{0}(S) \rightarrow H^{0}\left(\operatorname{sg} . X ; \mathbf{T}_{X}^{1}\right) \\
(\nu: \operatorname{spec}(\mathbb{C}[\varepsilon]) \rightarrow(S, 0)) \mapsto\left(\begin{array}{ccc}
\nu^{*}(\pi) & & \\
\downarrow & & \downarrow \pi \\
\downarrow & & \downarrow \\
\operatorname{spec}(\mathbb{C}[\varepsilon]) \stackrel{\nu}{\longrightarrow}(S, 0)
\end{array}\right)
\end{gathered}
$$

which is just restriction of the family $\pi$ to each first order tangent vector to $S$ at 0 .

(ii) The local coordinate expression for $\rho$ is as follows: if $v=\sum \alpha_{j} \partial / \partial t_{j} \in T_{0}(S)$, and $\mathbf{X}=\{F(x, t)=0\}=\left\{f(x)+\sum g_{j}(x) t_{j}+\cdots=0\right\}$, where $\left\{\partial / \partial t_{j}\right\}$ is the basis of $\left(\mathrm{m} / \mathrm{m}^{2}\right)^{*}$ dual to the basis $\left\{d t_{j}\right\}$ of $\mathrm{m} / \mathrm{m}^{2}$ defined by a minimal generating set $\left\{t_{j}\right\}$ for the maximal ideal $m$ of the point $0 \in S$, then

$$
\rho(v)=\sum \alpha_{j} g_{j}(x)=v \cdot \frac{\partial F}{\partial t}(x, 0)
$$

PROOF. Let $\nu$ be the morphism representing the vector $v=\sum \alpha_{j} \partial / \partial t_{j}$, so that $\nu$ corresponds to the local homomorphism sending $t_{j}$ to $\left(\alpha_{j} \cdot \varepsilon\right)$. Then pulling back the equation

$$
F(x, t)=f(x)+\sum g_{j}(x) t_{j}+\cdots
$$

by this morphism gives

$$
\nu^{*}(F)=f(x)+\left(\sum \alpha_{j} g_{j}(x)\right) \cdot \varepsilon
$$

so the first order deformation is $\sum \alpha_{j} g_{j}(x)$ as claimed. This is evidently linear in $\alpha$ Q.E.D.

COROllary (2.4). (i) The family $\pi: \mathbf{X} \rightarrow(S, 0)$ with $X=\pi^{-1}(0)$ gives rise to a natural linear system $\left|\mathbf{T}_{\pi}^{1}\right|$ on sg.X, namely the subsystem of $\left|\mathbf{T}_{X}^{1}\right|=$ $\mathbf{P}\left(H^{0}\left(\operatorname{sg} . X, \mathbf{T}_{X}^{1}\right)\right)$ defined by the image of the Kodaira-Spencer map, i.e.

$$
\left|\mathbf{T}_{\pi}^{l}\right|=\mathbf{P}\left(\rho\left(T_{0}(S)\right)\right) .
$$


(ii) If $T_{X}^{\mathrm{l}}$ is the vector space of (global) first order deformations of $X$, then there is a map $T_{X}^{\mathrm{l}} \rightarrow H^{0}\left(\mathrm{sg} . X, \mathbf{T}_{X}^{\mathrm{l}}\right)$ defining an intrinsic subsystem $\left|T_{X}^{\mathrm{l}}\right| \subseteq\left|\mathbf{T}_{X}^{\mathrm{l}}\right|$ which contains all the systems $\left|\mathbf{T}_{\pi}^{\mathrm{l}}\right|$ induced by families $\pi$ as above.

ProOF. (i) is clear.

(ii) $\left|T_{X}^{1}\right|$ is given by the image of the map defined by restricting global first order deformations to local ones, and the definition of $\rho$ for any family $\pi$ shows that $\rho$ factors through the image of this restriction map, i.e. $\nu^{*}(\pi) \in T_{X}^{1}$, by construction. Q.E.D.

REMARK (2.5). $\left|T_{X}^{1}\right|$ need not be all of $\left|\mathbf{T}_{X}^{1}\right|$, so (ii) gives a possibly nontrivial upper bound on $\left|\mathbf{T}_{\pi}^{1}\right|$. For example, consider a theta divisor $\Theta=\Theta(\Omega), \Omega \in \mathscr{H}_{g}$. The involution - id acts on $\Theta$ and hence on its first order deformation theory. The action on $T_{\Theta}^{1}$ is trivial since [S-V5] $T_{\Omega}\left(\mathscr{H}_{g}\right) \stackrel{\sim}{\rightarrow} T_{\Theta}^{1}$ for $g \geq 2$, so the image of $T_{\Theta}^{1} \rightarrow H^{0}\left(\mathbf{T}_{\Theta}^{1}\right)$ is contained in the subspace of sections fixed by the involution. But the action on $H^{0}\left(\mathbf{T}_{\Theta}^{1}\right)$ may be nontrivial: for the theta divisor of a generic genus 4 curve, $\mathbf{T}_{\Theta}^{1}$ is a skyscraper sheaf at 2 points which are interchanged by the involution.

Next we give the (generalized) criterion of Andreotti-Mayer for persistence of singularities.

DEFinition (2.6). Given $\pi: \mathbf{X} \rightarrow(S, 0)$ a family of hypersurfaces, and $p \in$ $\mathbf{C} \subseteq \mathbf{X}$ a critical point on $X=\pi^{-1}(0)$, we say that the singularity $p$ deforms, or persists, in the direction $[v] \in \mathbf{P} T_{0}(S)$, in the family $\pi$, if there exists a local curve $\tilde{\sigma}:(\Delta, 0) \rightarrow(\mathbf{C}, p)$ such that the composition $\sigma=(\pi \circ \tilde{\sigma}):(\Delta, 0) \rightarrow(D, 0)$ has tangent direction $[v]$ at 0 . Here $\Delta$ is a small disc in $\mathbb{C}^{1}$ centered at the origin.

Recall that the tangent line to a (local) curve at a point (which may be singular) is the unique line in the Zariski tangent space such that any hyperplane containing it has higher order of contact with the curve at that point than does a general hyperplane through the point. In local coordinates, the parametrized curve given by $\sigma(t)=\left(\alpha_{1} t^{r}+\cdots, \ldots, \alpha_{m} t^{r}+\cdots\right)$, where some $\alpha_{i} \neq 0$, has tangent vector $\left(\alpha_{1}, \ldots, \alpha_{m}\right)$ at $\sigma(0)$.

Proposition (2.7). If $\pi: \mathbf{X} \rightarrow S$ is a family of hypersurfaces, $X=\pi^{-1}(0)$, $p \in \operatorname{sg} . X, v \in T_{0}(S)$, and if $p$ persists in the direction $[v]$, then $\rho(v)(p)=0$, where $\rho(v) \in H^{0}\left(\right.$ sg. $\left.X, \mathbf{T}_{X}^{\mathrm{l}}\right)$.

Proof. Let $\mathbf{X}=\{F(x, t)=0\}$ locally, where $F=f(x)+\sum g_{j}(x) t_{j}+$ higher terms in the $t_{j}$ 's. Let $\sigma:(\Delta, 0) \rightarrow(D, 0)$ be the curve such that $p$ persists over it, and pull back the family $\sigma$. This gives us

$$
\begin{aligned}
& \sigma^{*}(\pi)=\left\{F^{*}(x, t)=0\right\} \subseteq \mathbb{C}^{m} \times \Delta \\
& \downarrow \\
& \Delta
\end{aligned}
$$

where $F^{*}(x, t)=f(x)+\left(\sum \alpha_{j} g_{j}(x)\right) t^{r}+h(x, t) \cdot t^{r+1}$. Now introduce the lift $\tilde{\sigma}$ and differentiate $F^{*}(\tilde{\sigma}(t))$ with respect to $t$ :

$$
0=\left(\frac{d}{d t}\right) F^{*}(\tilde{\sigma}(t))=\underbrace{\sum_{1}^{m}\left(\frac{\partial F^{*}}{\partial x_{i}}\right)(\tilde{\sigma}(t)) \cdot \tilde{\sigma}_{i}^{\prime}(t)}_{\|}+\left(\frac{\partial F^{*}}{\partial t}\right)(\tilde{\sigma}(t)) \cdot 1,
$$


where $\tilde{\sigma}(t)=\left(\tilde{\sigma}_{1}(t), \ldots, \tilde{\sigma}_{m}(t), t\right)$. Thus

$$
0=r \cdot\left(\sum_{1}^{m} \alpha_{j} g_{j}\left(\tilde{\sigma}_{i}(t)\right)\right) \cdot t^{r-1}+\text { higher terms in } t .
$$

Since this expression vanishes identically in $t$, the lowest order coefficient vanishes too, so we have in particular,

$$
\sum_{1}^{m} \alpha_{j} g_{j}\left(\tilde{\sigma}_{i}(0)\right)=v \cdot\left(\frac{\partial F}{\partial t}\right)(p)=\rho(v)(p)=0,
$$

by our earlier computation of the Kodaira-Spencer map. Q.E.D.

3. The Gauss map. Next we study the projective rational map of sg. $X$ defined by the family $\pi: \mathbf{X} \rightarrow S$.

DEFinition (3.1). The Kodaira-Spencer map $\rho: T_{0}(S) \rightarrow H^{0}\left(\operatorname{sg} . X_{0}, \mathbf{T}_{X_{0}}^{1}\right)$ from $\S 2$ defines a subspace $\rho\left(T_{0}(S)\right)$ of global sections of the line bundle $\mathbf{T}_{X_{0}}^{1}$, hence a rational map $\phi$ to $\mathbb{P} T_{0}^{*}(S)$,

$$
\phi: \operatorname{sg} . X_{0} \rightarrow \mathbb{P} \rho\left(T_{0}(S)\right)^{*} \stackrel{\rho^{*}}{\rightarrow} \mathbb{P} T_{0}^{*}(S)
$$

defined on the complement of the base locus of the space of sections $\rho\left(T_{0}(S)\right)$. $\phi=\phi_{\pi}$ is called the Gauss map of the deformation $\pi$ of $X_{0}$. As a map on points, $\phi(p)=(\partial F / \partial t)(p)$, which represents the hyperplane $\left\{v \in T_{0}(S): \rho(v)(p)=0\right\}$.

Proposition (3.2). Assume the base space $S$ of the family $\pi$ is smooth. Then $\phi$ has the following properties:

(1) $\phi$ is defined at $p \Leftrightarrow \mathbf{X}$ is smooth at $p$, and in that case, $\phi(p)=\operatorname{image}\left(\pi_{*, p}\right) \subseteq$ $T_{0}(S)$, where $\pi_{*, p}: T_{p} \mathbf{X} \rightarrow T_{\pi(p)}(S)$ is the derivative of $\pi$ at $p$.

(2) Assuming $\phi$ is defined at $p, \phi$ is unramified at $p \Leftrightarrow \mathbf{C}$ is smooth at $p$, and of the expected codimension in $\mathbf{X}$, i.e. of codimension $1+\operatorname{dim}_{p}(\mathbf{X})-\operatorname{dim}_{0}(S)$.

(3) If $\phi$ is defined at $p$, and if $v \in T_{0}(S)$ is any direction vector such that $p$ deforms along $v$, then $v$ lies in the hyperplane $\phi(p)$.

PROOF OF (1). In local coordinates, $p$ is in the base locus of $\rho\left(T_{0}(s)\right) \Leftrightarrow$ $(\partial F / \partial t)(p)=0 \in T_{0}^{*}(S)$, and since $p \in \operatorname{sg} . X_{0}$, we have $(\partial F / \partial x)(p)=0$. Thus $\phi$ is defined at $p \Leftrightarrow(\partial F / \partial t)(p) \neq 0 \Leftrightarrow d F(p) \neq 0 \Leftrightarrow \mathbf{X}$ is smooth at $p$.

ProOF OF (2). Locally, $\mathbf{C}$ has equations $\left\{F=\partial F / \partial x_{1}=\cdots=\partial F / \partial x_{n}=0\right\}$, so $\mathbf{C}$ is smooth at $p$ of the expected codimension $n$ in $\mathbf{X} \Leftrightarrow$

$$
\operatorname{rank}\left(\begin{array}{cc}
(\partial F / \partial t)(p) & (\partial F / \partial x)(p) \\
\left(\partial^{2} F / \partial t \partial x\right)(p) & \left(\partial^{2} F / \partial x^{2}\right)(p)
\end{array}\right)=n+1,
$$

and, since $(\partial F / \partial x)(p)=0$, if and only if the system of linear equations in $(\alpha, w) \in$ $\mathbb{C} \times \mathbb{C}^{n}$ :

$$
\left(\begin{array}{c}
\alpha \cdot(\partial F / \partial t)(p)+{ }^{t} w \cdot\left(\partial^{2} F / \partial t \partial x\right)(p)=0 \\
{ }^{t} w \cdot\left(\partial^{2} F / \partial x^{2}\right)(p)=0
\end{array}\right)
$$

has only the trivial solution $\left(\alpha,{ }^{t} w\right)=(0,0)$. Now consider the second of these equations. Since sg. $X_{0}:\left\{\left.F\right|_{t=0}=0=\left.(\partial F / \partial x)\right|_{t=0}\right\} \subseteq \mathbb{C}^{n}$, we have

$$
w \in T_{p}\left(\text { sg. } X_{0}\right) \Leftrightarrow\left(\partial^{2} F / \partial x^{2}\right)(p) \cdot w=0 \Leftrightarrow{ }^{t} w \cdot\left(\partial^{2} F / \partial x^{2}\right)(p)=0 .
$$


Thus we must show $\phi$ is unramified at $p \Leftrightarrow$ the only solution of the first equation with $w \in T_{p}\left(\operatorname{sg} . X_{0}\right)$ is $\left(\alpha,{ }^{t} w\right)=(0,0)$. So consider, in a neighborhood of $p$, the diagram of maps:

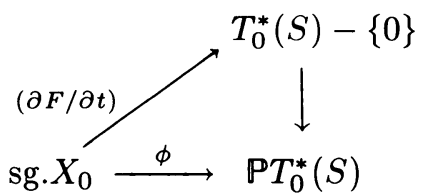

and their derivatives:

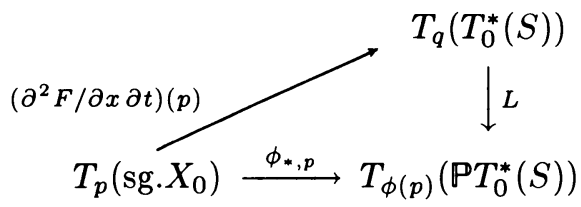

where $q=(\partial F / \partial t)(p)$. Since $\phi$ is defined at $p,(\partial F / \partial t)(p) \neq 0$, and the kernel of $L$ is the line $\{s \cdot(\partial F / \partial t)(p)\}$. Hence $\phi$ is unramified at $p \Leftrightarrow \phi_{*, p}$ is injective $\Leftrightarrow$ the only $w \in T_{p}\left(\operatorname{sg} . X_{0}\right)$ such that $\left(\partial^{2} F / \partial x \partial t\right)(p) \cdot w$ is in the kernel of $L$, is $w=0$. This, finally, is equivalent to saying the only solution of $\alpha \cdot(\partial F / \partial t)(p)+$ ${ }^{t} w \cdot\left(\partial^{2} F / \partial t \partial x\right)(p)=0$ with $w \in T_{p}\left(\operatorname{sg} . X_{0}\right)$ is $\left(\alpha,{ }^{t} w\right)=(0,0)$. Q.E.D.

PROOF OF (3). This is simply a restatement of the criterion of Andreotti-Mayer proved above.

Corollary (3.3). Let $\pi: \mathbf{X} \rightarrow S$ be as in Proposition (3.2), with $\pi$ proper on $\mathbf{C}$, or at least on sg. $X_{0}$.

(1) If $\mathbf{T}_{X_{0}}^{1}$ is ample on $\operatorname{sg} . X_{0}$ and $\operatorname{dim} S \leq \operatorname{dim} \operatorname{sg} . X_{0}$, then $\mathbf{X}$ is singular.

(2) Assuming $\mathbf{X}$ is smooth, if $\mathbf{C}$ is smooth of the expected codimension in $\mathbf{X}$, then $\mathbf{T}_{X_{0}}^{\mathrm{l}}$ is ample on $\mathrm{sg} . X_{0}$.

ProOF. For (1), if $m=\operatorname{dim} S \leq \operatorname{dimsg} . X_{0}$, any $m$ sections of an ample line bundle on sg. $X_{0}$ have a nonempty base locus [Ha1, p. 2] so, by $(3.2(1)), \mathrm{X}$ is singular. For (2), since $\phi: \operatorname{sg} . X_{0} \rightarrow \mathbb{P} T_{0}^{*}(S)$ is proper and, by $(3.2(2))$, unramified, it is a finite map so $\mathbf{T}_{X_{0}}^{1} \cong \phi^{*}(0(1))$ is ample [Gra, $\S 3.4$; Ha1, Chapter I, Proposition 4.4].

Next we compute the map $\phi$ in our two classical examples.

EXAMPLE (i). If $X \subseteq \mathbb{P}^{n}$ is a projective hypersurface of degree $d$, then

$$
\mathbf{T}_{X}^{1}=\left(N_{X / \mathbf{P}^{n}}\right)_{\mid \mathbf{s g} . X}=\left(\mathbf{O}_{\mathbf{P}^{n}}(d)\right)_{\mid \mathbf{s g} . X},
$$

and the map $\phi: \operatorname{sg} . X \rightarrow \mathbb{P} T_{X}^{*}(\mathbb{P}(n, d))$ is the restriction of the degree $d$ Veronese map $\mathbb{P}^{n} \rightarrow \mathbb{P}^{N(d)}$, with image in the hyperplane defined in $X$. To see this, recall that the linear system defining the Veronese map is given by the image of the restriction $H^{0}\left(\mathbf{P}^{n} ; \mathbf{O}(d)\right) \rightarrow H^{0}\left(\operatorname{sg} . X_{0} ; \mathbf{O}(d)\right)$, and that $H^{0}(\mathbf{P} ; \mathbf{O}(d)) \rightarrow H^{0}\left(X_{0} ; \mathbf{O}(d)\right)$ is surjective. Hence it suffices to check the commutativity of the diagram:

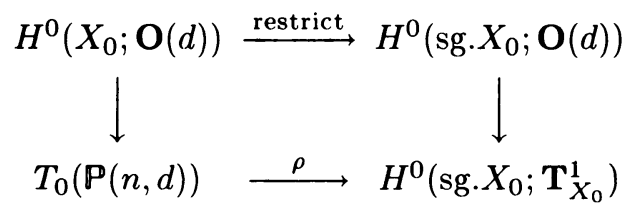


Now

$$
\begin{aligned}
T_{0}(\mathbb{P}(n, d)) & =\operatorname{Hom}\left(\mathbb{C} \cdot f_{0}, H^{0}\left(\mathbb{P}^{n} ; \mathbf{O}(d)\right) / \mathbf{C} \cdot f_{0}\right) \\
& =\operatorname{Hom}\left(\mathbb{C} \cdot f_{0}, H^{0}\left(X_{0} ; \mathbf{O}(d)\right)\right) \cong H^{0}\left(X_{0} ; \mathbf{O}(d)\right) .
\end{aligned}
$$

So let $g \in H^{0}\left(X_{0} ; \mathbf{O}(d)\right)$ be represented by the local functions $\left\{g_{\alpha}\right\}$ in terms of some affine open cover of $X_{0}$. Then tracing around the diagram in either direction yields the section of $H^{0}\left(\mathrm{sg} . X_{0}, \mathbf{T}_{X_{0}}^{1}\right)$ represented by $\left\{f_{\alpha}+\varepsilon \cdot g_{\alpha}\right\}$, where $\left\{f_{\alpha}\right\}$ is the family of local representatives of the equation $f_{0}$ for $X_{0}$.

EXAMPLE (ii). In the case of the universal theta divisor we have, at the point $\Omega \in \mathscr{H}_{g}$ corresponding to the p.p.a.v. $\left(A_{\Omega}, \Theta_{\Omega}\right)$,

$$
\begin{gathered}
\phi: \text { sg. } \Theta_{\Omega} \rightarrow \mathbb{P} T_{\Omega}^{*}\left(\mathscr{H}_{g}\right) \cong \mathbb{P} \operatorname{Sym}^{2} T_{0}^{*}\left(A_{\Omega}\right) \\
p \mapsto \phi(p)=(\partial \theta / \partial \Omega)(p) \mapsto\left(\partial^{2} \theta / \partial z^{2}\right)(p)
\end{gathered}
$$

using the heat equation. Here, $\phi$ is defined at $p \Leftrightarrow p$ is a double point of $\Theta$, and then $\phi(p)=$ the quadric tangent cone to $\Theta$ at $p$. In this setting, the original AndreottiMayer (incidence) criterion says that a double point $p \in \Theta$ can only persist along directions $v \in T_{\Omega} \mathscr{H}_{g}$ which belong to the hyperplane whose equation corresponds to the quadric tangent cone to $\Theta$ at $p$. Dually, each direction $v$ determines a hyperplane section of $\phi(\operatorname{sg} . \Theta)$ whose inverse image on sg. $\Theta$ contains all those double points which could possibly deform in the direction $v$. As we will see in the next section however, in fact only those points lying over points of tangency of the hyperplane section will actually deform along $v$.

Now consider the theta divisor of a nonhyperelliptic genus $g$ curve $C$. We want to relate our viewpoint to M. Green's solution of the "rank four quadrics problem" [Gre]. We see from the remarks above, that if we define $\bar{\rho}: T_{\Omega}\left(\mathscr{H}_{g}\right) \rightarrow$ $H^{0}\left((\operatorname{sg} . \Theta)_{\text {red. }} ;\left(\mathbf{T}_{\Theta}^{1}\right)_{\mid(\text {sg. } \Theta)_{\text {red. }}}\right)$ then $T_{\Omega(C)}\left(\mathbf{J}_{g}\right) \subseteq \operatorname{ker}(\bar{\rho})$, where $\mathbf{J}_{g} \subseteq \mathscr{H}_{g}$ is the locus of jacobian matrices. Therefore we get a map

$$
\begin{aligned}
\bar{\rho}: I_{2}(C)^{*}=N_{\left(\mathbf{J}_{g} / \mathscr{H}_{g}\right), \Omega(C)} & \rightarrow H^{0}\left((\mathrm{sg} . \Theta)_{\mathrm{red} .} ; T_{\mid(\mathrm{sg} . \Theta)_{\text {red. }}}^{1}\right) \\
\lambda & \mapsto\left(p \mapsto \lambda\left(Q_{p}\right)\right)
\end{aligned}
$$

where $I_{2}(C)$ denotes the vector space of quadratic polynomials vanishing on the canonical model of the curve $C$, and $Q_{p}$ is the quadratic term of the Taylor series for $\theta$ at $p$. Now, $\operatorname{Ker}(\bar{\rho})=(\phi(\operatorname{sg} . \Theta))^{\perp} \subseteq I_{2}(C)^{*}$, so

$$
\begin{aligned}
& \phi(\text { sg. } \Theta) \text { spans } I_{2}(C) \Leftrightarrow \bar{\rho} \text { is injective } \\
& \Leftrightarrow \operatorname{im} .(\bar{\rho}) \text { has dimension }\left(\begin{array}{c}
g-2 \\
2
\end{array}\right) \\
& \Leftrightarrow \operatorname{dim} . \operatorname{span}\left\{\left(\frac{\partial \theta}{\partial \Omega_{i j}}\right)(p)\right\}=\left(\begin{array}{c}
g-2 \\
2
\end{array}\right)=\frac{1}{2}(g-2)(g-3), \\
& \quad \text { as sections of } T_{\mid(\text {sg. } \Theta)_{\text {red }}}^{1} \\
& =\operatorname{dim} . \operatorname{span}\left\{\partial^{2} \theta / \partial z^{2}\right\} \quad \text { as functions on the zero set of }\{\theta, \partial \theta / \partial z\} .
\end{aligned}
$$

M. Green computed the dimension by using the resolution of $\Theta(C)$ provided by the Abel-Jacobi map $\alpha: C^{(g-1)} \rightarrow \Theta(C)$, and G. Kempf's theorem that deformations of $C^{(g-1)}$ come from deformations of $C[\mathbf{K e 2}]$. 
It is not difficult to give a proof of the injectivity of $\bar{\rho}$ from the point of view of deformation theory of the theta divisor, again using Kempf's result. In fact, it follows from Welter's work [Wel] that $\bar{\rho}$ has the same interpretation in characteristic $p$ so we are able to prove [S-V5]: for any nonhyperelliptic curve $C$ over an algebraically closed field of arbitrary characteristic, $I_{2}(C)$ is spanned by tangent cones to double points of $\Theta(C)$.

Finally, consider a product p.p.a.v. $A \times B$. Then $\Theta_{A \times B}=\left(\Theta_{A} \times B\right) \cup\left(A \times \Theta_{B}\right)$ so sg. $\left(\Theta_{A \times B}\right)=\left(\right.$ sg. $\left.\left(\Theta_{A}\right) \times B\right) \cup\left(\Theta_{A} \times \Theta_{B}\right) \cup\left(A \times\right.$ sg. $\left.\left(\Theta_{B}\right)\right)$. The Gauss map of Example (ii) $\phi_{A \times B}$ : sg. $\left(\Theta_{A \times B}\right) \rightarrow \mathbb{P} T_{A \times B}^{*}\left(\mathscr{H}_{g+h}\right)$ breaks into 3 parts:

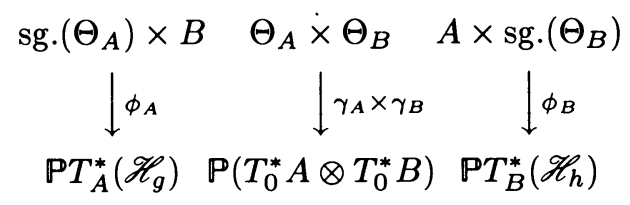

where $\gamma_{A} \times \gamma_{B}$ is the Segre product of the usual Gauss maps on the theta divisors $\Theta_{A}, \Theta_{B}$. To see this simply compute

$$
\frac{\partial^{2}}{\partial(z, w)^{2}}\left(\theta_{A} \theta_{B}\right)=\frac{\partial^{2}\left(\theta_{A}\right)}{\partial z^{2}} \theta_{B}+2 \frac{\partial\left(\theta_{A}\right)}{\partial z} \frac{\partial\left(\theta_{B}\right)}{\partial w}+\theta_{A} \frac{\partial^{2}\left(\theta_{B}\right)}{\partial w^{2}}
$$

in coordinates $(z, w)$, on $\mathbb{C}^{g} \times \mathbb{C}^{h}$ and use the decomposition

$$
\begin{aligned}
T_{A \times B}^{*}\left(\mathscr{H}_{g+h}\right) & \cong \operatorname{Sym}^{2} T_{0}^{*}(A \times B) \cong \operatorname{Sym}^{2} T_{0}^{*}(A) \oplus\left(T_{0}^{*}(A) \otimes T_{0}^{*}(B)\right) \oplus \operatorname{Sym}^{2} T_{0}^{*}(B) \\
& \cong T_{A}^{*}\left(\mathscr{H}_{g}\right) \oplus\left(T_{0}^{*}(A) \otimes T_{0}^{*}(B)\right) \oplus T_{B}^{*}\left(\mathscr{H}_{h}\right) .
\end{aligned}
$$

In particular, if $A$ is an abelian variety with nonsingular theta divisor $\Theta_{A}$ and $E$ is any elliptic curve, then sg. $\left(\Theta_{A \times E}\right) \cong \Theta_{A}$ and the Gauss map $\phi_{A \times E}$ is the usual Gauss map:

$$
\begin{array}{ll}
\Theta \stackrel{\gamma_{A}}{\rightarrow} \mathbb{P} T_{0}^{*}(A) \quad \text { followed by inclusion: } & \mathbb{P} T_{0}^{*}(A) \rightarrow \mathbb{P} T_{A \times E}^{*}\left(\mathscr{H}_{g+1}\right) . \\
x \mapsto\left[T_{x}\left(\Theta_{A}\right)\right] & {[h] \mapsto\left[h \otimes T_{0}^{*}(E)\right]}
\end{array}
$$

REMARK (3.4). Let $\mathscr{H}_{g, 1} \subset \mathscr{H}_{g+1}$ be the locus of products $A \times E$ where $A \in \mathscr{H}_{g}$, $E \in \mathscr{H}_{1}$. It is obvious that $\mathscr{H}_{g, 1} \subset \theta_{\text {null }}$, the locus of abelian varieties with a "vanishing theta null". From part (2) of Proposition (3.2), it can be deduced that also $\mathscr{H}_{g, 1} \subset \mathbf{N}_{0}^{*}$, the components of $\mathbf{N}_{0}$ in $\mathscr{H}_{g+1}$ residual to $\theta_{\text {null }}$. Then, from Theorem (4.1) it follows that the projectivized normal cone to $\mathscr{H}_{g, 1}$ in $\mathrm{N}_{0}^{*}$ at $A \times E$ contains the dual of the branch locus of the Gauss map $\gamma_{A}$ on $\Theta_{A}$. Details will be published elsewhere.

Before going on, observe that the Gauss maps $\phi_{s}$ : sg. $\left(X_{s}\right) \rightarrow \mathbb{P} T_{s}^{*}(S), s \in S$, fit together. That is, if $\pi: \mathbf{X} \rightarrow S$ is a family of hypersurfaces with $S$ smooth, then there is a rational map $\Phi: \mathbf{C} \rightarrow \mathbb{P} T^{*}(S)$ which is defined where $\mathbf{X}$ is smooth and, over $s \in S$, induces $\phi_{s}$. Indeed, as before, if $t$ denotes local coordinates on $S$ and $F$ is a local equation for $\mathbf{X}$, then $\Phi$ is given locally by $(\partial F / \partial t) d t$. (This can be expressed intrinsically as follows [Schl2, §3d; T1, pp. 14-15]: Since $S$ is smooth we have an exact sequence $\pi^{*} \mathbf{T}_{S}^{0} \rightarrow \mathbf{T}_{\mathbf{X} / S}^{1} \rightarrow \mathbf{T}_{\mathbf{X}}^{1} \rightarrow 0$. Here, since $\pi$ is a flat family of hypersurfaces, the relative $\mathbf{T}^{1}$-sheaf on $\mathbf{X}, \mathbf{T}_{\mathbf{X} / S}^{1}$, is a line bundle on $\mathbf{C}$ and, over $s \in S$, induces $\mathbf{T}_{X_{s}}^{1}$. Then, around a point of $\mathbf{C}$ which is smooth on $\mathbf{X}, \mathbf{T}_{\mathbf{X}}^{1}=0$, so $\Phi$ is determined by the universal mapping property [Ha2, Proposition 7.12, p. 162] of the projectivization of a vector bundle.) 
4. Tangent cones. Now we state and prove our main theorem.

THEOREM (4.1). Let $\pi: \mathbf{X} \rightarrow S$ be a family of varieties with only hypersurface singularities, let $\mathbf{C} \subseteq \mathbf{X}$ be the critical locus and assume the restriction $\eta$ of $\pi$ to $\mathrm{C}$ is proper. Let $D \subseteq S$ be the discriminant locus, $0 \in D$ a base point, and let $X=\pi^{-1}(0)$. Assume that $\mathbf{X}$ and $S$ are nonsingular. Let the map $\phi: \mathrm{sg} . X \rightarrow$ $\mathbb{P} T_{0}^{*}(S)$ be the Gauss map associated to the linear system $\left|\mathbf{T}_{\pi}^{1}\right|$, and defined by $\phi(p)=\operatorname{image}\left(\pi_{*, p}\right)$ and let the cycle sg.X be defined as follows: let $\sigma: \tilde{\mathbf{C}} \rightarrow \mathbf{C}$ be the blowup of the closed subscheme sg.X $\subseteq \mathrm{C}$, and let $\mathscr{E}=\sigma^{-1}(\mathrm{sg} . X)$ (the projectivized normal cone to sg. $X$ in $\mathbf{C})$. Then sg. $X=\sum_{\alpha} Z_{\alpha}$, where $\bigcup_{\alpha} \mathscr{E}_{\alpha}=$ $\mathscr{E}_{\text {red. }}$ i.e. $\mathscr{E}_{\alpha}$ are the irreducible components of the reduced set underlying $\mathscr{E}$, and $Z_{\alpha}=\sigma\left(\mathscr{E}_{\alpha}\right)$. Assume moreover, that $\phi$ is generically finite on each $Z_{\alpha}$ (a condition which is automatically satisfied whenever $\mathbf{C}$ is nonsingular and purely of the expected codimension in $\mathbf{X}$ by part (2) of Proposition (3.2)). Then, as sets,

$$
\mathbb{P} C_{0}(D)=\phi(\underline{\operatorname{sg} . X})^{*}
$$

i.e., $\mathbb{P} C_{0}(D)=\bigcup_{\alpha} \phi\left(Z_{\alpha}\right)^{*}$, as subsets of $\mathbb{P} T_{0}(S)$. (Here $V^{*}$ denotes the projective variety dual to $V$.)

PROOF. Consider the blowup diagram

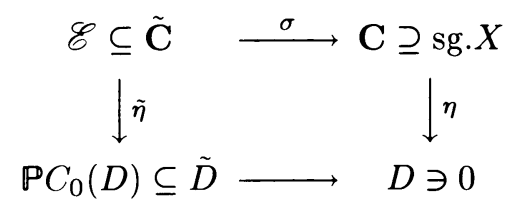

in which $\tilde{D}$ is the blowup of $D$ at 0 , with exceptional divisor $\mathbb{P} C_{0}(D)$, and $\tilde{\mathbf{C}}$ is the blowup of $\mathbf{C}$ along the scheme sg. $X$, with exceptional divisor $\mathscr{E}$. Since $\eta$ is proper and surjective, so is $\tilde{\eta}$, and since the components of $\mathscr{E}$ map via $\tilde{\eta}$ onto the set underlying $\mathbb{P} C_{0}(D)$, we have only to prove that, for each component $\mathscr{E}_{\alpha}$ of $\mathscr{E}_{\text {red. }}$, the two images $\phi\left(\sigma\left(\mathscr{E}_{\alpha}\right)\right)$ and $\tilde{\eta}\left(\mathscr{E}_{\alpha}\right)$ are mutually dual projective varieties. To put it more conveniently, consider the map

$$
\begin{gathered}
\phi \times 1:(\operatorname{sg} . X) \times\left(\mathbb{P} T_{0}(S)\right) \rightarrow\left(\mathbb{P} T_{0}^{*}(S)\right) \times\left(\mathbb{P} T_{0}(S)\right) \\
(p, v) \mapsto(\phi(p), v)
\end{gathered}
$$

and recall that by definition of blowing up $\mathbf{C}$ along sg. $X=\eta^{-1}(0)$, that $\mathscr{E} \subseteq$ $(\operatorname{sg} . X) \times\left(\mathbb{P} T_{0}(S)\right)$. Recall too, that a subvariety $\mathscr{F}^{r-1} \subseteq\left(\mathbb{P}^{r}\right)^{*} \times\left(\mathbb{P}^{r}\right)$ of dimension $r-1$, is a "conormal variety" (or dual correspondence) if and only if, for all $(H, v) \in$ $\mathscr{F}$, the conditions: (1) $v \in H$, and (2) $H$ is tangent to $\pi_{2}(\mathscr{F})$ at $v$ hold, where $\pi_{2}$ is the projection of $\mathscr{F}$ onto $\mathbb{P}^{r}$ and $H$ being tangent to $F$ at $v$ means $T_{v}(F) \subseteq H$ when $v$ is a nonsingular point of $F$, and, otherwise, that $(H, v)$ is a limit of such pairs. These conditions are referred to as the "incidence condition" and the "contact condition" respectively. Then the statement we want to prove becomes precisely that, for every component $\mathscr{E}_{\alpha}$ of $\mathscr{E}_{\text {red. }},(\phi \times 1)\left(\mathscr{E}_{\alpha}\right) \subseteq\left(\mathbb{P} T_{0}^{*}(S)\right) \times\left(\mathbb{P} T_{0}(S)\right)$ is a 
conormal variety. All the relevant data is contained in the following diagram:

$$
\begin{aligned}
& z=(p, v) \in \mathscr{E} \subseteq \tilde{\mathbf{C}} \quad \stackrel{\tilde{\Phi}}{\rightarrow} \overparen{\mathbb{P} T^{*}(S)} \supseteq\left(\mathbb{P} T_{0}^{*}(S)\right) \times\left(\mathbb{P} T_{0}(S)\right) \supseteq I \\
& \downarrow \tau \\
& \operatorname{sg} . X \subseteq \mathbf{C} \quad \stackrel{\Phi}{\rightarrow} \mathbb{P} T^{*}(S) \supseteq \mathbb{P} T_{0}^{*}(S) \\
& \downarrow \eta \quad \downarrow \nu \\
& 0 \in D \stackrel{\subseteq}{\leftrightarrows} S \ni 0
\end{aligned}
$$

Here, $\eta$ is the restriction of the original family $\pi: \mathbf{X} \rightarrow S, \nu$ is the projection of the cotangent bundle over $S, \sigma$ is the blowup of $\mathbf{C}$ along sg. $X=\eta^{-1}(0), \tau$ is the blowup of $\mathbb{P} T^{*}(S)$ along $\mathbb{P}_{0}^{*}(S)=\nu^{-1}(0), \Phi$ is the global Gauss map $\Phi(p)=\operatorname{image}\left(\pi_{*, p}\right)$, and $\tilde{\Phi}$ is the map induced by the universal property of blowing up, (i.e. since the lower square commutes, $\tilde{\Phi}$ exists uniquely making the upper square commute too). $\mathscr{E}$ and $\left(\mathbb{P} T_{0}^{*}(S)\right) \times\left(\mathbb{P} T_{0}(S)\right)$ are the exceptional divisors of the two blowups, and $I$ is the "incidence subvariety" consisting of pairs $(H, v)$ such that $v \in H$. If $(\xi, t)$ are coordinates on $\left(\mathbb{P} T_{0}^{*}(S)\right) \times\left(\mathbb{P} T_{0}(S)\right)$ let $\omega_{I}$ denote the "contact form" defined as $\xi \cdot d t$. Now we must check three assertions:

Claim (4.2). (i) For each point $z=(p, v) \in \mathscr{E}, \tilde{\Phi}(z)=(\phi(p), v)$; i.e. $\tilde{\Phi}_{\mid \mathscr{E}}=\phi \times 1$.

(ii) $\tilde{\Phi}\left(\mathscr{E}_{\text {red. }}\right) \subset I$ (incidence condition).

(iii) $\tilde{\Phi}^{*}\left(\omega_{I}\right) \equiv 0$, on the nonsingular points of $\mathscr{E}_{\text {red. }}$ (contact condition).

PROOF OF (i) AND (ii). Since $S$ is smooth we may consider it, locally near 0 , as an open set in $\mathbb{C}^{m}$, and the map $\pi$ as defining a rational map from a neighborhood of sg. $X$ in $\mathbf{C}$, to $\mathbb{P} T_{0}(S)$, by taking $p \in \mathbf{C}-\operatorname{sg} . X$ to the line joining $\pi(p)$ to 0 . Then $\tilde{\mathbf{C}}$ is defined set-theoretically as the closure of the graph of this map in (sg. $X) \times\left(\mathbb{P} T_{0}(S)\right)$. Now let $z=(p, v) \in \mathscr{E} \subset \tilde{\mathbf{C}}$ be any point on the exceptional divisor of this blowup, and let $\tilde{\alpha}:(\Delta, 0) \rightarrow(\tilde{\mathbf{C}}, z)$ be a local curve, with $\tilde{\alpha}(\Delta-0) \subseteq \tilde{\mathbf{C}}-\mathscr{E}$, and $\tilde{\alpha}(0)=z$. It follows that $v$ is the tangent direction of the curve $(\pi \circ \sigma \circ \tilde{\alpha})$ in $D$, and that via the curve $(\sigma \circ \tilde{\alpha})$ the singular point $p$ deforms in the direction $v$. Conversely if $p \in \operatorname{sg} . X$ does deform in some direction $v \in \mathbb{P} C_{0}(D)$, there is a curve $\alpha:(\Delta, 0) \rightarrow(\mathbf{C}, p)$ such that $v$ is the tangent direction of $(\pi \circ \alpha)$, and consequently the unique lift of $\alpha$ into $\tilde{\mathbf{C}}, \tilde{\alpha}:(\Delta, 0) \rightarrow \tilde{\mathbf{C}}$, has $\tilde{\alpha}(0)=(p, v)$. Thus, as a set, $\mathscr{E}=\left\{\right.$ those pairs $(p, v) \in(\operatorname{sg} . X) \times\left(\mathbb{P} C_{0}(D)\right)$ such that $p$ deforms in the direction $v$. Applying the same discussion to the blowup $\tau$, and noting that the diagram is commutative, we see that $\tilde{\Phi}(p, v)=(\phi(p), v)$. That is, to evaluate the "Grothendieck derivative" $\tilde{\Phi}$ on a point $(p, v)=z$ of the projectivized normal cone $\mathscr{E}$, just represent $z$ by an arc $\alpha:(\Delta, 0) \rightarrow(\mathbf{C}, p)$, compose the arc with $\Phi$, and then $\tilde{\Phi}(z)$ is the point (of the projectivized normal cone to $\mathbb{P} T_{0}^{*}(S)$ in $\mathbb{P} T^{*}(S)$ ) represented by the composite arc $(\Phi \circ \alpha):(\Delta, 0) \rightarrow\left(\mathbb{P} T^{*}(S), 0\right)$. This is the exact generalization of one definition of the Fréchet derivation common in differential geometry. Now by using the Andreotti-Mayer criterion, we have for all $(p, v) \in \mathscr{E}$, that $v \in \phi(p)$, and hence $\tilde{\Phi}\left(\mathscr{E}_{\text {red. }}\right) \subseteq I$.

ProOF OF (iii). First we prove that, as sets, $\Phi(\mathbf{C}) \subseteq \hat{D}=$ closure of the set of tangent hyperplanes at smooth points of $D=$ the "Nash blowup" of $D$ in $\operatorname{PT}^{*}(S)$. (Here each irreducible component of $\mathbf{C}$, even if it maps to a proper subvariety of an irreducible component of $D$, defines an irreducible component of $\hat{D}$.) That is, by Sard's theorem there is a dense open subset of points $p \in \mathbf{C}$ for which $\pi(p)$ is 
smooth on $D$ and

$$
T_{\pi(p)}(D)=\operatorname{image}\left(\eta_{*, p}\right) \subseteq \operatorname{image}\left(\pi_{*, p}\right)=\Phi(p) .
$$

Therefore, by continuity, $\Phi(\mathbf{C}) \subseteq \hat{D}$. (Let us remark that it is convenient to consider the reduced cycle $\underline{\mathbf{C}}$ defined by the irreducible components of $\mathbf{C}$, omitting any that are contained in $\eta^{-1}(0)=$ sg. $X$ or that miss $\eta^{-1}(0)$. Then with the corresponding cycle $\underline{D}=\eta(\underline{\mathbf{C}})$, we have $\Phi(\underline{\mathbf{C}})=\underline{\hat{D}}$. Indeed, since $\phi$ is generically finite on each $Z_{\alpha}$, $\Phi$ is generically finite on $\underline{\mathbf{C}}$ so each irreducible component of $\Phi(\underline{\mathbf{C}})$ has dimension $\geq \operatorname{dim}(S)-1$, which is the dimension of each irreducible component of $\underline{\hat{D}}$. Hence the containment $\Phi(\underline{\mathbf{C}}) \subseteq \underline{\hat{D}}$ is an equality. We note also that, whenever $\mathbf{C}$ is nonsingular and purely of the expected codimension in $\mathbf{X}, \Phi$ is finite on all of $\mathbf{C}$ so $\Phi(\mathbf{C})=\hat{D}$.) Now we want to use the fact that conormal varieties are characterized by the vanishing of the "contact form" [A1, Appendix 4, p. 367, Kl1, pp. 164-165]. So let $\omega$ be the contact form on $\mathbb{P}^{*}(S)$ and $\tilde{\omega}=\tau^{*}(\omega)$ the pull-back to $\overparen{\mathbb{P} T^{*}(S)}$. Since $\hat{D}$ is a conormal variety, we have that $\sigma^{*}\left(\Phi^{*}(\omega)\right)=\tilde{\Phi}^{*}(\tilde{\omega}) \equiv 0$, on $\tilde{\mathbf{C}}$, i.e. at nonsingular points of $\tilde{\mathbf{C}}_{\text {red. }}$. Let $\mathscr{E}_{\alpha}$ be any component of $\mathscr{E}_{\text {red. }}$. Normalizing $\tilde{\mathbf{C}}$ if necessary, choose a point $z \in \mathscr{E}_{\alpha}$ such that $z$ is smooth both on $\tilde{\mathbf{C}}$ and on $\mathscr{E}_{\text {red. }}$.

LEMMA (4.3). If $\omega_{I}$ is the contact form on $I$, and if $\{f=0\}$ is a local equation for $\left(\mathbb{P} T_{0}^{*}(S)\right) \times\left(\mathbb{P} T_{0}(S)\right)$ in $\overparen{\mathbb{P} T^{*}(S)}$, near $\tilde{\Phi}(z)$, then locally $d \tilde{\omega}=\omega_{1} \wedge d f+f \beta$, for some two-form $\beta$.

Proof. Choose local coordinates $\left(t_{1}, \ldots, t_{m}\right)$ on $S$ and homogeneous fiber coordinates $\left(\xi_{1}, \ldots, \xi_{m}\right)$ on $\mathbb{P} T^{*}(S)$, and blow up along the scheme $\mathbb{P} T_{0}^{*}(S)$ defined by the ideal $\left(t_{1}, \ldots, t_{m}\right)$. In nonhomogeneous coordinates, where say $\xi_{1} \neq 0$, the blowing down map $\tau$ is given by

$$
\tau\left(t_{1}, u_{2}, \ldots u_{m} ; \xi_{2}, \ldots, \xi_{m}\right)=\left(t_{1}, t_{1} u_{2}, \ldots, t_{1} u_{m} ; \xi_{2}, \ldots, \xi_{m}\right)
$$

where $t_{i}=t_{1} u_{i}, i=2, \ldots, m$. Now in these local (nonhomogeneous) coordinates the contact form on $\mathbb{P} T^{*}(S)$ is given by $\omega=d t_{1}+\sum_{2}^{m} \xi_{j} d t_{j}$, and thus

$$
\tau^{*}(\omega)=\tilde{\omega}=d t_{1}+\sum_{2}^{m} \xi_{j} d\left(t_{1} u_{j}\right) .
$$

Therefore,

$$
\begin{aligned}
d \tilde{\omega} & =d\left(d t_{1}\right)+d\left(\sum_{2}^{m} \xi_{j} d\left(t_{1} u_{j}\right)\right)=\sum_{2}^{m} d \xi_{j} \wedge d\left(t_{1} u_{j}\right) \\
& =\sum_{2}^{m} t_{1} d \xi_{j} \wedge d u_{j}+\sum_{2}^{m} u_{j} d \xi_{j} \wedge d t_{1} \\
& =t_{1} \cdot\left(\sum_{j=2}^{m} d \xi_{j} \wedge d u_{j}\right)+\left(\sum_{j=2}^{m} u_{j} d \xi_{j}\right) \wedge d t_{1} \\
& =f \cdot \beta+\omega_{I} \wedge d f,
\end{aligned}
$$

since $t_{1}=0$ is the local equation of the exceptional divisor of $\tau$. Q.E.D. Lemma.

Consequently, we have the following equations holding on $\tilde{\mathbf{C}}$ locally near $z$ :

$$
0 \equiv d\left(\tilde{\Phi}^{*}(\tilde{\omega})\right)=\tilde{\Phi}^{*}(d \tilde{\omega})=\tilde{\Phi}^{*}\left(f \beta+\omega_{I} \wedge d f\right)=g^{n} \cdot \tilde{\beta}+\tilde{\Phi}^{*}\left(\omega_{I}\right) \wedge d g^{n}
$$


where $\{g=0\}$ is a local equation for $\mathscr{E}_{\text {red. }} \subseteq \tilde{\mathbf{C}}$ near $z$. Factoring out $g^{n-1}$ leaves

$$
0 \equiv g \cdot \tilde{\beta}+\tilde{\Phi}^{*}\left(\omega_{I}\right) \wedge n d g \text { on } \tilde{\mathbf{C}} \text { near } z \text {. }
$$

Now let $w \in T_{z} \mathscr{E}_{\text {red. }}$ be any tangent vector, and $v \in T_{z} \tilde{\mathbf{C}}$ be any vector such that $v \notin T_{z} \mathscr{E}_{\text {red. }}$, and evaluate the previous two-form on the pair $(v, w)$. Since $g(z)=0$, and $d g$ vanishes precisely on $T_{z}\left(\mathscr{E}_{\text {red. }}\right)$, we get $0=\left(\tilde{\Phi}^{*}\left(\omega_{I}\right)(w)\right) \cdot(d g(v))$, and since $v \notin T_{z}\left(\mathscr{E}_{\text {red. }}\right), d g(v) \neq 0$ implies $\tilde{\Phi}\left(\omega_{I}\right)(w)=0$. Hence $\tilde{\Phi}^{*}\left(\omega_{I}\right) \equiv 0$ on $\mathscr{E}_{\text {red. as }}$ claimed, and so, in fact, $\omega_{I} \equiv 0$ on $\tilde{\Phi}\left(\mathscr{C}_{\text {red. }}\right)$. Note that, even if normalization of $\tilde{\mathbf{C}}$ is necessary, this same conclusion is valid since the restriction of the normalization map to any irreducible component of the reduced preimage of $\mathscr{E}_{\text {red. }}$ is, at a generic point, unramified to a nonsingular point of $\mathscr{E}_{\text {red. }}$. Last of all we remark that since $\phi$ is generically finite on each $Z_{\alpha}, \tilde{\Phi}$ is generically finite on each $\mathscr{E}_{\alpha}$, so $\tilde{\Phi}\left(\mathscr{C}_{\text {red. }}\right)$ has pure dimension $=\operatorname{dim} .\left(\mathbb{P} T_{0}(S)\right)-1$. It therefore follows that $\tilde{\Phi}\left(\mathscr{E}_{\text {red. }}\right)$ is indeed a union of conormal varieties. Q.E.D. Claim and Theorem.

Explicitly, here are the answers provided by Theorem (4.1) and proof to the questions posed in $\S 1$ :

2. A given point $p \in \operatorname{sg} . X_{0}$ deforms in those directions at $0 \in S$, i.e. points of $\mathbf{P} T_{0}(S)$, for which the corresponding hyperplane of $\mathbb{P} T_{0}^{*}(S)$ is tangent at $p$ to some $Z_{\alpha}$. (This means $p$ is nonsingular on $Z_{\alpha}, \phi$ is unramified on $Z_{\alpha}$ at $p, \phi(p)$ is nonsingular on $\phi\left(Z_{\alpha}\right)$, and the hyperplane contains the tangent space to $\phi\left(Z_{\alpha}\right)$ at $\phi(p)$, or, is a limit, for points of $Z_{\alpha}$ near $p$, of such hyperplanes.)

3. The smoothing directions, i.e. the directions in which no singularity deforms, are those for which the corresponding hyperplane of $\mathbb{P} T_{0}^{*}(S)$ is transversal, i.e. nowhere tangent, to each $\phi\left(Z_{\alpha}\right)$.

1. If any irreducible component of $\mathrm{sg} . X_{0}$ is an irreducible component of $\mathbf{C}$, there are no directions in which all singular points of $X_{0}$ deform. Otherwise, all singular points of $X_{0}$ deform along those directions for which the corresponding hyperplane of $\mathrm{PT}_{0}^{*}(S)$ contains $\phi\left(\mathrm{sg} . X_{0}\right)$. This is because all the irreducible components of sg. $X_{0}$ will occur among $\left\{Z_{\alpha}\right\}$ (see $\S 5$ ) and a hyperplane is tangent at every point of $\phi\left(Z_{\alpha}\right) \Leftrightarrow$ it contains $\phi\left(Z_{\alpha}\right)$. Thus, the set of directions in which all singularities deform is $\mathbb{P}(\operatorname{ker} \bar{\rho})$ where $\bar{\rho}: T_{0}(S) \rightarrow H^{0}\left(\mathbf{T}_{X_{0} \mid\left(\mathbf{s g} . X_{0}\right)_{\text {red }}}^{1}\right)$, and no tangency condition is required to state answer (1)!

Now that we have seen that the set of all tangent directions to $D$, at the point corresponding to $X$, is dual to a reduced space underlying the scheme sg. $X$ via $\phi$, it is natural to ask for the geometric meaning of the directions dual to the actual scheme sg. $X$; i.e., which tangent directions to $D$ correspond to hyperplanes which contain (via $\phi$ ) the Zariski tangent spaces at points of the scheme sg. $X$ ? Our result is that, if $\mathbf{C}$ is nonsingular along sg. $X$, these correspond to the "immersive directions" in $C_{X}(D)$, i.e. those which can be represented as tangents to smoothly immersed arcs in $D$. To prove it, reconsider the map $\eta: \mathbf{C} \rightarrow D$, and let $\xi \in \mathbf{C}$ be a nonsingular point and $0=\eta(\xi) \in D$. Then it is readily seen that the set of immersive tangent directions to $D$ at 0 in which $\xi$ persists as a singular point, is precisely $\eta_{*}\left(T_{\xi} \mathbf{C}\right)$, the image of the tangent space to $\mathbf{C}$ at $\xi$ under the Fréchet derivative. Thus we will prove the following:

Proposition (4.4). Given $\pi: \mathbf{X} \rightarrow S$ as above, with $\mathbf{X}$ and $S$ smooth, let $\xi \in \mathbf{C}$ be any point, $0=\pi(\xi) \in D$, and $X=\pi^{-1}(0)$. If we denote by $\eta: \mathbf{C} \rightarrow D$ 
the restriction of $\pi$ to $\mathbf{C}$, then we have

$$
\text { image }\left(\eta_{*, \xi}\right)=\left(T_{\xi}(\phi(\text { sg. } X))\right)^{*} \quad \text { in } \mathbb{P} T_{0}(S) \text {. }
$$

Here, $T_{\xi}(\phi(\operatorname{sg} . X))$ denotes $d \phi\left(T_{\xi}(\operatorname{sg} . X)\right)$, realized as a linear subspace of $\mathbb{P} T_{0}^{*}(S)$. (If $\mathbf{C}$ is nonsingular of the expected codimension in $\mathbf{X}, \phi$ is unramified so $T_{\xi}(\phi(\operatorname{sg} . X))$ may be thought of as the Zariski tangent space to $\phi(\mathrm{sg} . X)$ along the "branch" determined by $\xi$.)

PROOF. In local coordinates, if $\xi=(x, v)$ then $\pi(x, v)=v$, so

$$
\operatorname{image}\left(\eta_{*, \xi}\right)=\left\{v \in T_{0}(S):(\exists x)\left((x, v) \in T_{\xi} \mathbf{C}\right)\right\}
$$

and if $\mathbf{X}=\{F=0\}$ locally, then

$$
T_{\xi} \mathbf{C}=\operatorname{ker}\left[\begin{array}{cc}
{[0]} & {\left[\frac{\partial F}{\partial v}(\xi)\right]} \\
{\left[\frac{\partial^{2} F}{\partial x^{2}}(\xi)\right]} & t^{t}\left[\frac{\partial}{\partial x}\left(\frac{\partial F}{\partial v}\right)\right]
\end{array}\right]
$$

Hence,

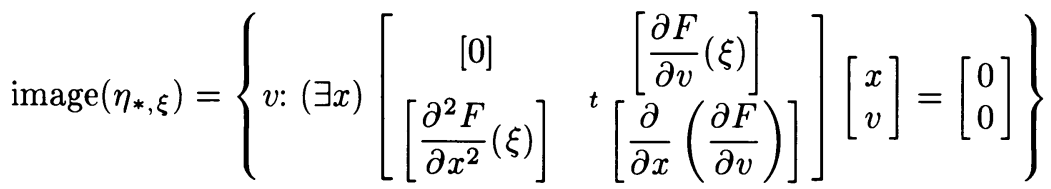

$$
\begin{aligned}
& =\left\{v:(1)(v \perp \phi(\xi)), \text { and }(2)(\exists x)\left(x \cdot \frac{\partial^{2} F}{\partial x^{2}}(\xi)+v \cdot \frac{\partial^{2} F}{\partial x \partial t}=0\right)\right\} \\
& =\left\{v:(1) \text { holds, and }(2)(\exists x)\left(\frac{\partial^{2} F}{\partial x^{2}}(\xi) \cdot x=-{ }^{t}(d \phi)(v)\right)\right\} \\
& =\left\{v:(1) \text { holds, and (2) }{ }^{t}(d \phi)(v) \in \text { image }\left(\frac{\partial^{2} F}{\partial x^{2}}(\xi)\right)\right\}
\end{aligned}
$$

(where the matrix $\partial^{2} F(\xi) / \partial x^{2}$ is thought of as a linear transformation)$$
=\left\{v:(1) \text { holds, and }(2)^{t}(d \phi)(v) \perp \operatorname{ker}\left(\frac{\partial^{2} F}{\partial x^{2}}(\xi)\right)\right\}
$$$$
=\left\{v \text { : (1) holds, and (2) }{ }^{t}(d \phi)(v) \perp T_{\xi}(\operatorname{sg} . X)\right\}
$$$$
\left.=\left\{v:(1) \text { holds, and }(2)(d \phi)\left(T_{\xi}\right)(\operatorname{sg} . X)\right) \perp v\right\} \text {. Q.E.D. }
$$

When $\xi \in \mathbf{C}$ is a singular point, this proposition is little help with the tangent cone to the discriminant, for, $\operatorname{im}\left(\eta_{*}, \xi\right)$ in $\mathbb{P} T_{0}(S)$ need not be contained in $\mathbb{P} C_{0}(D)$ and, since $\phi$ ramifies at $\xi, T_{\xi}(\phi(\mathrm{sg} . X))$ may be smaller than expected. If $\mathbf{C}$ is nonsingular along sg. $X$ and, in addition, sg. $X$ is nonsingular, then all directions in $C_{X}(D)$ are immersive so we recover the duality theorem.

COROllary (4.5). Given $\pi: \mathbf{X} \rightarrow S$ as above, with $\mathbf{X}$ and $S$ smooth, assume $\mathbf{C}$ is smooth of the expected codimension in $\mathbf{X}$. Let $0 \in D$ be a base point, $X=$ $\pi^{-1}(0)$, and assume sg.X is smooth. Then, as sets,

$$
\mathbb{P} C_{0}(D)=\phi(\operatorname{sg} \cdot X)^{*} \text {. }
$$

PROOF. As sets,

$$
\mathbb{P} C_{0}(D)=\bigcup_{\xi \in \text { sg. } X} \operatorname{im}\left(\eta_{*, \xi}\right)=\bigcup_{\xi} T_{\xi}(\phi(\operatorname{sg} . X))^{*}=\phi(\operatorname{sg} . X)^{*} .
$$


The first equality holds since $\eta: \mathbf{C} \rightarrow D$ is proper and surjective, and $\mathbf{C}$ and $\eta^{-1}(0)=$ sg. $X$ are assumed smooth (cf. $\S 6$ ). The second equality holds by the proposition and the third since sg. $X$ is smooth and $\phi$ is unramified by part (2) of (3.2). Q.E.D.

REMARK (4.6) (NORMAL CONES). While Theorem (4.1) describes the tangent cones to the discriminant, we may want to know instead the normal cone to the discriminant along some given subvariety $E$, e.g. (i) "locally trivial" deformations or (ii) deformations with the same dimensional singular locus. Without going back to the proof, we get the result at a generic point $s$ of $E$ from the comparison: $\left.\mathbb{P} C_{E}(D)\right|_{s}=\mathbb{P}\left(C_{s}(D) / T_{s}(E)\right)$, or equivalently, $\mathbb{P} C_{s}(D)$ is a cone over $\left.\mathbb{P} C_{E}(D)\right|_{s}$ with vertex $\mathbb{P} T_{s}(E)$. It is elementary to check this when $D$ has pure codimension 1 in (smooth) $S$. Namely, take any smooth point of $E$ and use coordinates $\underline{u}, \underline{v}$ on $S$ so that $v=0$ is $E$. Expand out the equation for $D, \delta(u, v)=a_{\underline{k}}(u) v^{\underline{k}}+$ $a_{k+1}(u) v \underline{k+1}+\cdots$ where $a_{\underline{r}}(u) v^{\underline{r}}$ is homogeneous of degree $r$ in $v$ and $a_{\underline{k}}(u)$ is not identically 0 on $E$. Then, at points of $E$ where $a_{\underline{k}}$ is not 0 , the stated comparison holds. In general the comparison of tangent and normal cones is valid where $D$ is normally flat along $E$ [Hi, Chapter II, Proposition 1].

REMARK (4.7) (SINGUlaR TOTAL SPACE). The same methods are applicable when the total space $\mathbf{X}$ is singular. Namely assuming $S$ is nonsingular and no irreducible component of $\mathbf{C}$ is contained in $\operatorname{sg}$. $(\mathbf{X})$, first blow up $\mathbf{C}$ along the base locus of $\Phi$ to get a morphism $\mathbf{C}^{\prime} \stackrel{\Phi^{\prime}}{\rightarrow} \mathbb{P} T^{*}(S)$ and replace sg. $X$ by its total transform. Then the formulation and proof of Theorem (4.1) can be copied.

5. Distinguished subvarieties. We keep the notation from Theorem (4.1) and the assumption that $\mathbf{X}$ and $S$ are nonsingular.

DEFINITION (5.1). The varieties $\left\{Z_{\alpha}\right\}$ are called the distinguished subvarieties [Fu, p. 95] or generalized components of $\mathrm{sg} . X$. They are defined in terms of the inclusion of $\mathrm{sg} . X$ in $\mathbf{C}$.

If $Z \subseteq \operatorname{sg} . X$ is a distinguished subvariety and $\phi$ is generically finite on $Z, \phi(Z)^{*}$ may only be a "distinguished subvariety" of $\mathbb{P} C_{0}(D)$ rather than an irreducible component. However, if $\phi(Z)^{*}$ is a hypersurface of $\mathbb{P} T_{0}(S)$, it must be an irreducible component of $\mathbb{P} C_{0}(D)$.

LEMMA (5.2). If $Z \subseteq \mathrm{sg} . X_{0}$ is a distinguished subvariety and $\phi$ is generically finite on $Z$ then there is a unique irreducible component of $\mathscr{E}$ over $Z$.

PROOF. Suppose $\mathscr{E}^{\prime}$ and $\mathscr{E}^{\prime \prime}$ are irreducible components of the exceptional divisor which map onto $Z$. Then $\mathscr{E}^{\prime}$ and $\mathscr{E}^{\prime \prime}$ are contained in $\mathbb{P} T_{0}(S) \times Z$ and $\tilde{\phi}\left(\mathscr{E}^{\prime}\right)=\tilde{\phi}\left(\mathscr{E}^{\prime \prime}\right)$ in $\mathbb{P} T_{0}(S) \times \mathbb{P} T_{0}^{*}(S)$ is the dual correspondence $\mathscr{F}$ of $\phi(Z)$. Since $\phi$ is generically finite on $Z$, there exists a complex ball $U$, nonempty and open, in $Z$ such that $\phi$ induces an isomorphism from $U$ to a smooth, nonempty open subset $\phi(U)$ of $\phi(Z)$. But now $\mathscr{E}^{\prime} \cap\left(\mathbb{P} T_{0}(S) \times U\right)$ and $\mathscr{E}^{\prime \prime} \cap\left(\mathbb{P} T_{0}(S) \times U\right)$ both map isomorphically to $\mathscr{F} \cap\left(\mathbb{P} T_{0}(S) \times \phi(U)\right)$ by $\tilde{\phi}$, hence coincide. Since $\mathscr{E}^{\prime}$ and $\mathscr{E}^{\prime \prime}$ have a nonempty open subset in common, they are equal.

Now consider the problem of determining the distinguished subvarieties.

LEMMA (5.3). An irreducible component of sg.X is a distinguished subvariety if and only if it is not an irreducible component of $\mathbf{C}$. 
ProOF. First, note that an irreducible component $Z$ of $\operatorname{sg} . X$ is a distinguished subvariety $\Leftrightarrow Z \subseteq \sigma(\tilde{\mathbf{C}})$, where $\sigma: \tilde{\mathbf{C}} \rightarrow \mathbf{C}$ is the blowing-up of $\mathbf{C}$ with center sg. $X$. If $Z$ is an irreducible component of $\mathbf{C}$ then at a generic point of $Z$ the ideal of sg. $X$ in $\mathbf{C}$ is nilpotent so the blowup is empty there and $Z \nsubseteq \sigma(\tilde{\mathbf{C}})$. If $Z$ is not an irreducible component of $\mathbf{C}$, then $Z$ is contained in an irreducible component of $\mathbf{C}$ which is not contained in the center of the blowing-up, and $\sigma(\tilde{\mathbf{C}})$ contains any such irreducible component [Fu, B.6.5; H-R, §1], so $Z \subseteq \sigma(\tilde{\mathbf{C}})$.

REMARKS (5.4). (1) When the critical locus $\mathbf{C}$ is known to have the expected dimension, it is obvious whether an irreducible component of $\mathrm{sg} . X$ is an irreducible component of $\mathbf{C}$, namely, if and only if it has dimension $=\operatorname{dim}(S)-1$.

(2) If $\mathbf{T}_{X}^{1}$ is ample on sg. $X$ then $\mathbf{C}$ has the expected dimension (in a neighborhood of sg.X). Indeed, the Gauss map $\Phi: \mathbf{C} \rightarrow \mathbb{P} T^{*}(S)$ will be finite (over a neighborhood of $0 \in S$ ) and send an irreducible component of $\mathbf{C}$ onto the conormal variety of its image under $\pi$ in $S$ (see the proof of Theorem (4.1)). But a conormal variety in $\mathbb{P} T^{*}(S)$ has dimension $=\operatorname{dim}(S)-1$.

(3) If $Z$ is an irreducible component of $\operatorname{sg} . X$ and $\phi(Z)=\mathbb{P} T_{0}^{*}(S)$, then $Z$ is an irreducible component of $\mathbf{C}$. This is because if $Z$ were an irreducible component of sg. $X$ but not of $\mathbf{C}$, there would be an irreducible component $\mathscr{E}$ of $\widetilde{\mathbf{C}}$ mapping onto $Z$ (by Lemma (5.3)) so consider $\tilde{\phi}(\mathscr{E}) \subseteq \mathbb{P} T_{0}^{*}(S) \times \mathbb{P} T_{0}(S)$. The projection to $\mathbb{P} T_{0}(S)$ is, in any case, contained in $\phi(Z)^{*}$ (by the proof of Theorem (4.1)) so $\phi(Z) \neq \mathbb{P} T_{0}^{*}(S)$.

EXAMPLES (5.5). (1) A given family $\pi: \mathbf{X} \rightarrow S$ determines two others as follows: $\pi_{1}: \mathbf{X} \times \mathbb{P}^{1} \rightarrow S, \pi_{1}(x, z)=\pi(x)$ (product of the hypersurfaces $\left\{X_{s}\right\}$ with $\mathbb{P}^{1}$ ). The Gauss map is $\phi_{\pi_{1}}: \operatorname{sg} .(X) \times \mathbb{P}^{1} \rightarrow X \stackrel{\phi_{\pi}}{\rightarrow} \mathbb{P} T_{0}^{*}(S)$.

$\pi_{2}: \mathbf{X} \times \Delta \rightarrow S \times \Delta, \pi_{2}(x, z)=(\pi(x), z), \Delta=$ unit disc in $\mathbb{C}$ (base change of $\pi$ by $S \times \Delta \rightarrow S,(t, z) \mapsto t)$. The Gauss map is $\phi_{\pi_{2}}: \operatorname{sg} . X \stackrel{\phi_{\pi}}{\rightarrow} \mathbb{P} T_{0}^{*}(S) \hookrightarrow$ $\mathbb{P} T_{0}^{*}(S \times \Delta)$. Suppose the critical locus of $\pi$ has the expected dimension and has an irreducible component over 0 . Then the critical locus of $\pi_{1}$ does not have the expected dimension and has an irreducible component over 0 while the critical locus of $\pi_{2}$ has the expected dimension and does not have an irreducible component over $(0,0)$. In particular, starting with $\pi: \mathbf{X}=\mathbb{C}^{2} \rightarrow \mathbb{C}=S,(x, y) \mapsto x y=t$, illustrates the various phenomena.

(2) Here is an example to show the converse is false in Remarks (5.4)(2) and (3). Let $Y_{0}$ be a curve in $\mathbb{P}^{2}$ with a node at a point $p$ and consider a generic net $\Pi$ (2-parameter linear family of curves) through $Y_{0}$. Then, blowing up $\mathbb{P}^{2}$ at $p$ and taking the total transform of the net gives a family $\pi: \mathbf{X} \rightarrow \Pi$ (cf. Example (7.1)) in which $X_{0}$ is the proper transform of $Y_{0}$ plus twice the exceptional line. The reader may check that $\mathbf{C}$ has the expected dimension but $\phi_{\pi}$ maps $\left(\mathrm{sg} . X_{0}\right)_{\text {red. }}$ to a point. ( $\mathbf{C}$ has two irreducible components and is nonreduced everywhere along the exceptional line; sg. $X_{0}$ is equal to the exceptional line except at two points where it is nonreduced. All the hypotheses of Theorem (4.1) are satisfied.)

PROPOSITION (5.6). If $\mathbf{C}$ is nonsingular and has no component in $\mathrm{sg} . X$, then the distinguished subvarieties of $\mathrm{sg} . X$ are intrinsic to the analytic space $\mathrm{sg} . X$.

PROOF. We will check that for any closed (nowhere dense) analytic subspace $Y$ of a smooth analytic space $V$, the distinguished subvarieties of $Y$ (with respect 
to $Y \subset V$, i.e. the images in $Y$ of the irreducible components of the exceptional divisor of the blowup of $V$ along $Y$ ) are intrinsic to $Y$. First, whether a subvariety $Z \subseteq Y$ is distinguished can be checked on any open neighborhood in $V$ of any point of $Z$ (if $\mathbf{U}$ is such a neighborhood, check whether there is an irreducible component of the inverse image of $U \cap Z$ of the required dimension $(=\operatorname{dim}(V)-1)$ mapping onto an irreducible component of $\mathrm{U} \cap Z)$. Now, given $p \in Y$, there is an embedding $(Y, p) \subseteq\left(V_{0}, p\right)$ in a smooth germ (i.e. "embed $(Y, p)$ in its Zariski tangent space at $p$ ") s.t. every (closed) embedding $(Y, p) \rightarrow\left(V^{\prime}, p\right)$ in a smooth germ is obtained as a composition $(Y, p) \subseteq\left(V_{0}, p\right) \rightarrow\left(V^{\prime}, p\right)$. Then $(V, p) \cong\left(V_{0}, p\right) \times\left(\Delta_{k}, 0\right)$ where $\Delta_{k}$ is a $k$-dimensional polydisc $(k \geq 0)$. Therefore, it suffices to compare $Y \subseteq V_{0}$ with $Y \times\{0\} \subseteq V_{0} \times \Delta_{k}$ and since $\Delta_{k}=\Delta^{k}$ it suffices by induction to consider $k=1$. Then it is elementary to check that $\mathbb{P} C_{Y \times\{0\}}\left(V_{0} \times \Delta\right) \cong \mathbb{P}\left(C_{Y}\left(V_{0}\right) \oplus 1\right)[\mathbf{F u}, \mathbf{p}$. 87,2 nd paragraph], i.e. the irreducible components in the exceptional divisor over $Y \times\{0\}$, are cones over the irreducible components in the exceptional divisor over $Y$, and have the same images in $Y \times\{0\} \cong Y$.

At this point, note that the Duality Theorem stated in the introduction follows immediately from Theorem (4.1), combined with (3.2)(2), (5.3), and (5.6). (There is one technicality in case $\operatorname{dim} S=\operatorname{dim}(\operatorname{sg} . X)+1$. Then, each irreducible component of sg. $X$ which does not occur as distinguished subvariety in (4.1) maps, by (3.2)(2), (isomorphically) onto $\mathbb{P} T_{0}^{*}(S)$ so the statement is still correct.)

For plane curves and normal crossing varieties we can identify the distinguished subvarieties of the singular locus, in the sense of Proposition (5.6).

Proposition (5.7). Let $X \subset \mathbb{P}^{2}$ be a plane curve. Then the set of distinguished subvarieties of sg.X consists precisely of (a) those irreducible components of $X_{\text {red. }}$ which are not reduced in $X$ and (b) the singular points of $X_{\text {red. }}$.

PrOOF. The subvarieties (a), which are the 1-dimensional irreducible components of sg. $X$, are obviously distinguished. Any other distinguished subvariety of sg. $X$ must be a point so it remains to show that the distinguished points of sg. $X$ are precisely (b). For this, consider a point $p$ of an affine plane curve $X$ defined by a polynomial $f(x, y)$. It suffices to show that the Jacobian ideal $J=\left(f, f_{x}, f_{y}\right)$ is locally principal at $p \Leftrightarrow p$ is a nonsingular point of $X_{\text {red. }}$. (Then, by Zariski's Main Theorem and the universal property of blowing-up, the blowup of $J$ will have a divisor over $p \in A^{2} \Leftrightarrow J$ is not locally principal at $p$.) If $p$ is nonsingular on $X_{\text {red. }}, f=g^{n}$ where, locally at $p,\left(g, g_{x}, g_{y}\right)$ is the unit ideal so $J=\left(g^{n}, n g^{n-1} g_{x}, n g^{n-1} g_{y}\right)=\left(g^{n-1}\right)$. For the converse, assume $p$ is singular on $X_{\text {red. }}$ and write $f=f_{1}^{n_{1}} \cdots f_{r}^{n_{r}}$ where $f_{1}, \ldots, f_{r}$ are the distinct irreducible factors. Since $\gamma=f_{1}^{n_{1}-1} \cdots f_{r}^{n_{r}-1}$ divides $f, f_{x}$, and $f_{y}$, we consider $I=J / \gamma$ and claim $p$ is an isolated point of $V(I)$, hence $I$ is not locally principal at $p$. Obviously $f / \gamma=f_{1} \cdots f_{r}$ vanishes at $p$ and, since either $r \geq 2$ or $r=1$ and $\partial f_{1} / \partial x, \partial f_{1} / \partial y$ both vanish at $p$,

$$
\frac{f_{x}}{\gamma}=\sum_{j=1}^{r} n_{j} f_{1} \cdots \frac{\partial f_{j}}{\partial x} \cdots f_{r}
$$

and

$$
\frac{f_{y}}{\gamma}=\sum_{j=1}^{r} n_{j} f_{1} \cdots \frac{\partial f_{j}}{\partial y} \cdots f_{r}
$$


both vanish at $p$; thus $p \in V(I)$. Since $V(I) \subseteq V\left(f_{1}\right) \cup \cdots \cup V\left(f_{r}\right)$, to show $p$ is isolated in $V(I)$ it will suffice to show that none of the irreducible curves $V\left(f_{i}\right)$ is contained in $V(I)$. If $V\left(f_{i}\right) \subseteq V(I)$ then $I \subseteq\left(f_{i}\right)$ since $\left(f_{i}\right)$ is prime. Then

$$
\begin{aligned}
& f_{i} \mid \sum_{j} n_{j} f_{1} \cdots \frac{\partial f_{j}}{\partial x} \cdots f_{r} \\
& \quad \text { and } f_{i} \mid \text { each term for } j \neq i \text { in the sum } \\
& \quad \Rightarrow f_{i}\left|f_{1} \cdots \frac{\partial f_{i}}{\partial x} \cdots f_{r} \Rightarrow f_{i}\right| \frac{\partial f_{i}}{\partial x}
\end{aligned}
$$

since $f_{1}, \ldots, f_{r}$ are distinct irreducibles. This implies, by taking degree in $x$, that $f_{i}$ is a polynomial in $y$ alone. Likewise, since $f_{i} \mid \sum n_{j} f_{1} \cdots \partial f_{j} / \partial y \cdots f_{r}, f_{i}$ is a polynomial in $x$ alone so $f$ is constant, contradiction.

REMARK (5.8). Proposition (5.7) can be deduced from Iverson [I, §6], which contains a similar argument. It completes the description of the set-theoretic tangent cone to the discriminant for curves of degree $d$ in $\mathbb{P}^{2}$ at an arbitrary point (establishing the "Proposed Generalization" on p. 3 of [S-V4]). Further discussion, including the multiplicities of the irreducible components, i.e., the scheme-theoretic tangent cone, will be published elsewhere.

Normal crossing varieties are an important type of variety with hypersurface singularities. These have local equation $x_{1} \cdots x_{k}=0$ in $\mathbb{C}^{n}$ where the value of $k$, $1 \leq k \leq n$, depends on the point and defines a stratification of the variety. The deformation theory of normal crossings has been studied by R. Friedman [Fr].

PROPOSITION (5.9). Let $X$ be a normal crossing variety. Then the distinguished subvarieties of $\mathrm{sg} . X$ are precisely the irreducible components of the closures of the standard strata.

PROOF. Locally, let $\Sigma_{k}$ be the stratum: $x_{1}=\cdots=x_{k}=0$ in $X: x_{1} \cdots x_{k}=0$, $k \geq 2$, in $\left(\mathbb{C}^{n}, 0\right)$. We must show that, in the blow-up of $\mathbb{C}^{n}$ along the ideal $\left(x_{2} \cdots x_{k}, \ldots, x_{1} \cdots x_{k-1}\right)$ of sg. $X$, an irreducible component of the exceptional divisor maps onto $\Sigma_{k}$. Since the variables $x_{k+1}, \ldots, x_{n}$ are missing, it evidently suffices to show that the blowup of $\mathbb{C}^{k}$ along this same ideal has an irreducible component of the exceptional divisor over 0 . To construct the blowup, use the generators of the ideal to define a rational map from $\mathbb{C}^{k}$ to $\mathbb{P}^{k-1}$ and take the closure of the graph in $\mathbb{C}^{k} \times \mathbb{P}^{k-1}$. We have to check that all of $\{0\} \times \mathbb{P}^{k-1}$ is in the closure. But, since the rational map $\left(x_{1}, \ldots, x_{k}\right) \mapsto\left(1 / x_{1}, \ldots, 1 / x_{k}\right)$ from $\mathbb{P} T_{0}\left(\mathbb{C}^{k}\right)$ to $\mathbb{P}^{k-1}$ is dominant, this is obvious.

REMARK (5.10). If $X$ is a normal crossing variety then it is elementary to check that $\mathrm{sg} . X$ is reduced, hence the only "associated subvarieties" of $\mathrm{sg} . X$ (in the sense of primary decomposition) are its irreducible components. On the other hand, whenever there are more than double crossings, sg. $X$ has distinguished subvarieties besides its irreducible components.

In this paper we have concentrated on the set-theoretic description of $\mathbb{P} C_{0}(D)$. When $D$ has pure codimension 1 in $S, \mathbb{P} C_{0}(D)$ is determined as subscheme of $\mathbb{P} T_{0}(S)$, by its set-theoretic irreducible components together with their multiplicities. Let us indicate how to compute the multiplicities.

PROPOSITION (5.11). Keep the notation and all the hypotheses of Theorem (4.1) and assume, further, that $\mathbf{C}$ is irreducible and generically finite over $D$. Let 
$r_{\alpha}$ be the "geometric multiplicity" of the irreducible components $\mathscr{E}_{\alpha}$ of $\mathscr{E}$, i.e. [ $[\mathscr{E}]=$ $\sum_{\alpha} r_{\alpha}\left[\mathscr{E}_{\alpha}\right]$ in the notation from Fulton $[\mathbf{F u}, 1.5]$. Let $\phi_{\alpha}=\left.\phi\right|_{Z_{\alpha}}$ and $V_{\alpha}=\phi\left(Z_{\alpha}\right)$. Then

$$
\sum_{\alpha}{ }^{\prime} r_{\alpha} \operatorname{deg}\left(\phi_{\alpha}\right)\left[V_{\alpha}^{*}\right]=\operatorname{deg}(\mathbf{C} / D)\left[\mathbb{P} C_{0}(D)\right],
$$

where the sum is over those $\alpha$ for which $V_{\alpha}^{*} \subseteq \mathbb{P} T_{0}(S)$ is a hypersurface. In particular,

$$
\operatorname{mult}_{0}(D)=\frac{1}{\operatorname{deg}(\mathbf{C} / D)} \sum_{\alpha}{ }^{\prime} r_{\alpha} \operatorname{deg}\left(\phi_{\alpha}\right) \operatorname{deg}\left(V_{\alpha}^{*}\right) .
$$

PROOF. The formula is obtained by applying the projection formula [Fu, Proposition $2.3\left(\right.$ c), p. 34] to the map $\tilde{\mathbf{C}} \stackrel{f}{\rightarrow} \tilde{D}$, i.e. since $\mathscr{E}=f^{*}\left(\mathbb{P} C_{0}(D)\right), g_{*}([\mathscr{E}])=$ $\operatorname{deg}(\mathbf{C} / D)\left[\mathbb{P} C_{0}(D)\right]$ where $g=\left.f\right|_{\mathscr{E}}$. On the other hand,

$$
g_{*}([\mathscr{E}])=\sum_{\alpha} r_{\alpha} g_{*}\left(\left[\mathscr{E}_{\alpha}\right]\right)=\sum_{\alpha}{ }^{\prime} r_{\alpha} \operatorname{deg}\left(\phi_{\alpha}\right)\left[V_{\alpha}^{*}\right]
$$

since

$$
\operatorname{deg}\left(\mathscr{E}_{\alpha} / V_{\alpha}^{*}\right)= \begin{cases}\operatorname{deg}\left(\phi_{\alpha}\right) & \text { if } V_{\alpha}^{*} \text { is a hypersurface, by (4.2)(i), } \\ 0 & \text { otherwise. }\end{cases}
$$

Now we recover the well-known description of the tangent cone to the discriminant for deformations of isolated hypersurface singularities.

Corollary (5.12) (Milnor, Brieskorn-Pham, Teissier [T1, §5; Chapter III, §4]). Let $(X, \Sigma)$ be the germ of a space $X$ at a finite set $\Sigma=(\operatorname{sg} . X)_{\text {red. }}$. of isolated hypersurface singularities and consider a versal deformation $\pi:(\mathbf{X}, \Sigma) \rightarrow$ $(S, 0)$ of $(X, \Sigma)$, i.e., assume the Kodaira-Spencer map $\rho: T_{0}(S) \rightarrow H^{0}\left(\mathrm{sg} . X, \mathbf{T}_{X}^{1}\right)$ is surjective, where $S$ is smooth. Then, as schemes, $\mathbb{P} C_{0}(D)=\bigcup_{p \in \Sigma} \mu_{p} H_{p}$, the union of distinct hyperplanes indexed by the singular points of $X$, each counted with multiplicity equal to the Milnor number of the corresponding isolated hypersurface singularity.

ProOF. Since $\Sigma=(\mathrm{sg} . X)_{\text {red. }}$ is finite, the restriction of $\pi$ to the critical locus $(\mathbf{C}, \Sigma)$ is proper $\left[\mathbf{M u 3},(3.11)\right.$, p. 44]. Since $\mathbf{T}_{X}^{1}$ is a line bundle on the finite, but not necessarily reduced, space sg. $X$ and $\rho$ is surjective, it is elementary to see that the Gauss map $\phi$ embeds sg. $X$ in $\mathbb{P} T_{0}^{*}(S)$. Therefore, $\mathbf{X}$ and $\mathbf{C}$ are smooth, by (3.2)(1) and (2), and the points $\phi(p) \in \mathbb{P} T_{0}^{*}(S), p \in \Sigma$, are distinct. Now set $H_{p}=\phi(p)^{*}$, the dual hyperplane, which is simply $\operatorname{ker}\left\{T_{0}(S) \rightarrow H^{0}\left(\left.\mathbf{T}_{X}^{1}\right|_{p}\right)\right\}$ in $\mathbb{P} T_{0}(S)$, and the set-theoretic statement follows from (4.1). To get the multiplicities, we apply, for each $p \in \Sigma,(5.11)$ to the family $(\mathbf{X}, p) \rightarrow(S, 0)$. The restriction of $\pi$ to $(\mathbf{C}, p)$ has degree one over its image $D_{p}$ since the Gauss map $\Phi$ is an isomorphism to the Nash blowup $\hat{D}_{p}$, which then maps birationally to $D_{p}$. Thus, by the proof of (5.6), we are reduced to showing: if $X=\{f=0\} \subset \mathbb{C}^{n}$ is a hypersurface having at 0 an isolated singularity with Milnor number $\mu$, then the exceptional divisor $\mathscr{E}$ over 0 in the blowup of $\mathbb{C}^{n}$ along sg. $X$ has one irreducible component and its geometric multiplicity is $\mu$. To blow up the Jacobian ideal $J=\left(f, f_{x_{1}}, \ldots, f_{x_{n}}\right)$, we take the closure in $\mathbb{C}^{n} \times \mathbb{P}^{n}$ of the graph of the rational map: $\mathbb{C}^{n} \rightarrow \mathbb{P}^{n}$, $x \mapsto\left(f(x), f_{x_{1}}(x), \ldots, f_{x_{n}}(x)\right)$. Now the integral dependence of $f$ on the ideal $\left(x_{1} f_{x_{1}}, \ldots, x_{n} f_{x_{n}}\right)$ [T2, Chapter $\left.0, \S 0.5(1)\right]$ shows that when $x \rightarrow 0$ along any 
arc in $\mathbb{C}^{n},\left(f(x), f_{x_{1}}(x), \ldots, f_{x_{n}}(x)\right) \rightarrow\left(0, \alpha_{1}, \ldots, \alpha_{n}\right) \in \mathbb{P}^{n}$ for some $\alpha_{1}, \ldots, \alpha_{n}$. Therefore $E=\mathscr{E}_{\text {red. }}$ is $\{0\} \times\left\{\alpha_{0}=0\right\}$. Thus $[\mathscr{E}]=r[E]$ and it remains to prove $\mu=r$.

$\mu=\operatorname{dim}_{\mathbb{C}} \mathbb{C}\left\{x_{1}, \ldots, x_{n}\right\} / I$, where $\mathbb{C}\left\{x_{1}, \ldots, x_{n}\right\}$ is the analytic local ring of $\mathbb{C}^{n}$ at 0 and $I=\left(f_{x_{1}}, \ldots, f_{x_{n}}\right)$

[Mi, Problem 3, p. 115; L, 5.11]

$=$ mult $I$ since $f_{x_{1}}, \ldots, f_{x_{n}}$ is a regular sequence [T2, Chapter $\left.0, \S 1.1\right]$

$=$ mult $J$ since $f$ is integral over $I$ [T2, Chapter $0, \S 1.1]$

$=\operatorname{deg} s_{0}\left(\operatorname{sg} .(X, 0), \mathbb{C}^{n}\right), \quad$ where $s_{0}=$ zeroth relative Segre class

[Fu, Examples 4.3.4, 19.2.5]

$=\operatorname{deg}\left(c_{1}\left(\mathbf{O}_{\mathscr{E}}(-\mathscr{E})\right)^{n-1} \cap[\mathscr{E}]\right), \quad$ where $c_{1}=$ first Chern class

[Fu, Corollary 4.2.2]

$=r \operatorname{deg}\left(c_{1}\left(\mathbf{O}_{\mathscr{E}}(-\mathscr{E})\right)^{n-1} \cap[E]\right)=r \quad$ since $E \cong \mathbf{P}^{n-1}$ and $\mathbf{O}_{E}(-\mathscr{E}) \cong \mathbf{O}_{\mathbf{P}^{n-1}}(1)$. Q.E.D

6. Tangent cones to a parametrized space. In this section we drop the hypothesis that the space in which we are computing the tangent cone is a discriminant locus, and introduce instead the concept of a generic exceptional fiber of a map, as follows:

Given a generically finite, proper, surjective map $f: X \rightarrow Y$, with $X$ nonsingular and $Y$ reduced, let $y \in Y$ be a point for which: $f^{-1}(y)$ is a nonsingular scheme, i.e.,

(1) $f^{-1}(y)$ is nonsingular as reduced space (possibly positive dimensional).

(2) The differential $f_{*}$ is injective on all fibers of the normal bundle $N$ to $f^{-1}(y)$.

Then, if we blow up $X$ along $f^{-1}(y)$ to get $\tilde{X}$, the composition $\tilde{X} \rightarrow X \rightarrow Y$ still satisfies (1), and (2), so that if we blow up $Y$ at $y$, getting $\tilde{Y}$, we have a lift of $f$ :

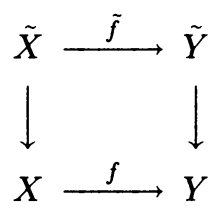

whose restriction to $\mathrm{P}(N)=$ projective normal bundle of $f^{-1}(y)$ in $X$, is given by the differential of the composition $\tilde{X} \rightarrow X \rightarrow Y$ [D-S, Chapter I, Lemma 3.1]. We denote it also by $f_{*}$. This restriction of $\tilde{f}$ to the exceptional fibers gives us a surjective map:

$$
f_{*}: \mathbb{P}(N) \rightarrow \mathbb{P}\left(C^{\prime}\right)=\mathbb{P}\left(\left(C_{y} Y\right)_{\text {red. }}\right)
$$

from the projective normal cone of $f^{-1}(y)$ in $X$ onto the reduced projective tangent cone of $Y$ at $y$.

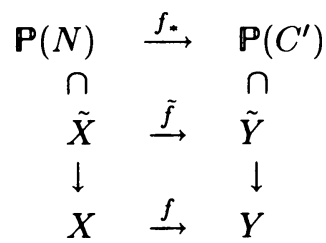


We want to relate the invariants of the two generically finite maps $f$ and $f_{*}$. For simplicity, assume $\mathbf{P}\left(C^{\prime}\right)$ is irreducible.

Since $\tilde{f}$ has injective differential on the normal line bundle to $\mathbf{P}(N) \subseteq \tilde{X}$, and $f_{*}: \mathbf{P}(N) \rightarrow \mathbf{P}\left(C^{\prime}\right)$ preserves dimension, the kernel of the differential of $f$ has been eliminated generically along the exceptional fiber by the blowing up process, so that $\tilde{f}$ has injective differential at a general point of every component of $\mathbf{P}(N) \subseteq \tilde{X}$. Therefore at a general point of $\mathbf{P}\left(C^{\prime}\right) \subseteq \tilde{Y}, \tilde{Y}$ has a smooth branch, and thus either $\tilde{Y}$ is smooth at a generic point of $\mathbb{P}\left(C^{\prime}\right)$ and such a point is a regular value of $\tilde{f}$, or else $\tilde{Y}$ is locally reducible generically along $\mathbb{P}\left(C^{\prime}\right)$. By factoring $\tilde{f}$ through the normalization of $\tilde{Y}$ if necessary, we can conclude:

$$
\begin{aligned}
\operatorname{deg}\left(f_{*}\right) \geq & \operatorname{deg}(f) \\
\text { and equality holds } \Leftrightarrow & \tilde{Y} \text { smooth at a generic } \\
& \text { point of } \mathbf{P}\left(C^{\prime}\right) \\
\Leftrightarrow & \tilde{Y} \text { normal at a generic } \\
& \text { point of } \mathbf{P}\left(C^{\prime}\right) .
\end{aligned}
$$

This formula can be refined as follows: Since $\mathbb{P}\left(C^{\prime}\right)$ is a projective variety in the projective Zariski tangent space of $Y$ at $y$, it has a tautological line bundle $\mathbf{O}(-1)$, and by the definition of $f_{*}$,

$$
\left(f_{*}\right)^{*}(\mathbf{O}(-1))=E=\text { the normal line bundle to } \mathbf{P}(N) \text { in } \tilde{X} .
$$

Thus $c_{1}(E)=\left(f_{*}\right)^{*} c_{1}(\mathbf{O}(-1))$, and if we raise both sides of this equation to the power $n=\operatorname{dim} \cdot \mathbb{P}(C)=\operatorname{dim} \cdot \mathbb{P}(N)$, and evaluate on the fundamental class of $\mathbf{P}(N)$, we have

$$
\begin{aligned}
\operatorname{deg} .\left\{(-1)^{n} c_{1}(E)^{n}[\mathbf{P}(N)]\right\} & \left.=\operatorname{deg}\left\{\left(f_{*}\right)^{*}\left(c_{1}(\mathbf{O}(1))^{n}\right)[\mathbf{P}(N))\right]\right\} \\
& =\operatorname{deg}\left(f_{*}\right) \cdot \operatorname{deg}\left(\mathbf{P}\left(C^{\prime}\right)\right) .
\end{aligned}
$$

(If $\mathbf{P}\left(C^{\prime}\right)$ is reducible, the last product is a dot product, summed over the components of $\mathbb{P}\left(C^{\prime}\right)$.)

On the other hand, if we apply Fulton's and MacPherson's formula [Fu, p. 74] involving the Segre class of $E$ to the composition $\tilde{X} \rightarrow X \rightarrow Y$, since $\tilde{X} \rightarrow X$ is birational it gives us

$$
\operatorname{deg}\left\{s(E)_{n}[\mathbf{P}(N)]\right\}=\operatorname{deg}(f) \cdot \operatorname{deg}(C) .
$$

Since

$$
c(E)=1+c_{1}(E)
$$

and

$$
s(E)=c(E)^{-1}=1-c_{1}(E)+\cdots+(-1)^{n} c_{1}^{n}(E),
$$

we have $s(E)_{n}=(-1)^{n} c_{1}^{n}(E)$, so that the left sides of the two formulas agree, and we get

Degree Formula (6.1). $\operatorname{deg}(f) \cdot \operatorname{deg}(C)=\operatorname{deg}\left(f_{*}\right) \cdot \operatorname{deg}\left(C^{\prime}\right)$ and thus $\operatorname{deg}(f)=$ $\operatorname{deg}\left(f_{*}\right)$ if and only if the top dimensional cycle of $C$ has multiplicity one. 
EXAMPLE (6.2). A simple example, the normalization of a tacnode, pointed out by Cornalba, illustrates clearly the relation between $\operatorname{deg}(f)$ and $\operatorname{deg}\left(f_{*}\right)$ :

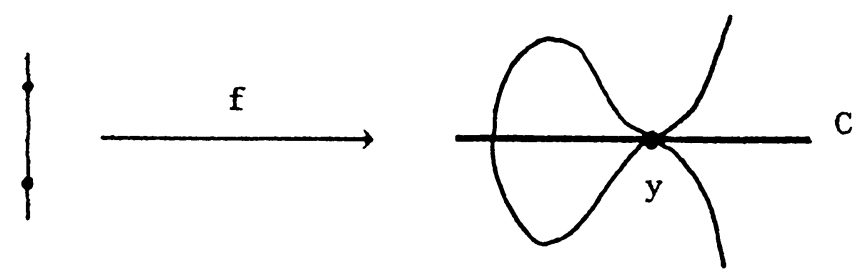

$C$ is a double line, $\operatorname{deg}(f)=\operatorname{deg}\left(C^{\prime}\right)=1$, and $\operatorname{deg}\left(f_{*}\right)=\operatorname{deg}(C)=2$.

EXAMPLE (6.3). One application of the degree formula is to refine the geometric argument given in Griffiths-Harris [G-H] for Riemann's singularities theorem. Consider the Abel-Jacobi map $C^{(g-1)} \stackrel{\alpha}{\rightarrow} \Theta \subseteq J(C)$ parametrizing the theta divisor. If $L \in \Theta$ is any point, then $\alpha^{-1}(L) \cong|L|=\mathbb{P}^{r}$, where $r=h^{0}(L)-1$, by Abel's theorem and its converse so the reduced fibers of $\alpha$ are smooth. By Mattuck-Mayer's version of the Riemann-Roch theorem [M-M, p. 230], at each point of $\alpha^{-1}(L)$, the derivative $\alpha_{*}$ has an $r$-dimensional kernel, so the fibers are actually smooth as schemes and the hypotheses of this section are verified. It follows that the image under $\alpha_{*}$ of the normal bundle in $C^{(g-1)}$ to $\alpha^{-1}(L)$ is the tangent cone to $\Theta$ at $L$, at least as a set.

This set, a hypersurface in $\mathbb{P}^{g-1}$, is computed in [G-H, pp. 343-345]. To see that this is the tangent cone as a scheme as well, one only needs to compute that the multiplicity of the fundamental cycle is one. But by the argument in [G-H, pp. 345-346], $\alpha_{*}$ has degree one on the normal bundle to $\alpha^{-1}(L)$. Hence by our formula (6.1), the tangent cone is reduced as a scheme. For related methods, see [Ke1; Fu, Example 4.3.2].

EXAMPLE (6.4). The tangent cone to o $\in \Theta(W)$, where $W_{3} \subseteq \mathbb{P}^{4}$ is a smooth cubic threefold, can be computed the same way. This time the parametrizing map is, by Clemens-Griffiths [C-G, Theorem 13.4], the difference map $F \times F \stackrel{\delta}{\rightarrow} J(W)$ from the Cartesian product of two copies of the Fano surface $F$ of lines on $W$. Now $\delta$ collapses the diagonal $\Delta \cong F$ to the (unique) singular point $0 \in \Theta(W)$. The projectivized normal bundle to $\Delta$ in $F \times F$ is isomorphic to the projectivized tangent bundle of $F$, which is [C-G, Proposition 12.9] the tautological $\mathbb{P}^{1}$-bundle over $F \subseteq \operatorname{Gr}(1,4)$. Moreover their argument shows [C-G, 12.4] that the AbelJacobi map $F \rightarrow J(W)$ is an embedding whose derivative at $L \in F$ embeds the projective line $\mathbb{P}\left(T_{L} F\right)$ onto the line $L$ in $\mathbb{P} T_{0}(J(W)) \cong \mathbb{P}^{4}$.

Hence $\delta_{*}$ is injective on the normal bundle $N$ to $\Delta$ in $F \times F$, and maps the projectivized normal bundle $\mathbb{P} N$ onto the union of the lines parametrized by $F$ in $\mathbb{P} T_{0} J(W)=\mathbb{P}^{4}$, i.e. onto $W_{3} \subseteq \mathbb{P}^{4}$. Hence $\mathbb{P} C_{0} \Theta(W)$ is $W_{3}$ at least as sets and to see it as schemes, it suffices by $(6.1)$ to check that $\operatorname{deg} \delta=\operatorname{deg} \delta_{*}$. But by [C-G, p. 348 ], $\operatorname{deg} \delta=6$, while $\operatorname{deg} \delta_{*}=$ the number of lines on $W$ through a general point, which is also 6. Similar arguments are given in [B; Fu, p. 80].

The possibility of using the normal bundle along the fibers to compute the tangent cone to the image of a map was emphasized by A. Mayer in the 1960s. To see the role of this method and to review and compare with $\S 4$, consider the map $\eta: \mathbf{C} \rightarrow D \subseteq S$ from critical locus to discriminant, and a point $0 \in D$. The induced 
map on blowups $\tilde{\eta}: \tilde{\mathbf{C}} \rightarrow \tilde{D}$ restricts to $g: \mathscr{E}=\mathbb{P} C_{\text {sg. } X_{0}}(\mathbf{C}) \rightarrow \mathbb{P} C_{0}(D) \subseteq \mathbb{P} T_{0}(S)$ on exceptional divisors. When $\mathbf{C}$ and $\eta^{-1}(0)=$ sg. $X_{0}$ are both smooth, then $\mathscr{E}=$ PN, the projectivized normal bundle and $g=\eta_{*}$, the ordinary "Fréchet derivative". In general, even if $\mathbf{C}$ is smooth, it is insufficient to know $\eta_{*, \xi}: T_{\xi}(\mathbf{C}) \rightarrow T_{0}(S)$ for $\xi \in \eta^{-1}(0)$ : if $\left.p \in \mathscr{E}\right|_{\xi}, g(p)$ is the limiting tangent along the (possibly singular) image of any arc in $\mathbf{C}$ "through $p$ ". Still, $g: \mathscr{E} \rightarrow \mathbb{P} T_{0}(S)$ is defined by sections of the line bundle $\mathbf{O}_{\mathscr{E}}(-\mathscr{E})$; on the other hand, there is the line bundle $\sigma^{*}\left(\mathbf{T}_{X_{0}}^{1}\right)$ on $\mathscr{E}$ and the composed map: $\mathscr{E} \stackrel{\sigma}{\rightarrow} \operatorname{sg} . X_{0} \stackrel{\phi}{\rightarrow} \mathbb{P} T_{0}^{*}(S)$. Our main theorem is that, on $\mathscr{E}_{\text {red. }}, g$ and $\phi \circ \sigma$ have projectively dual images; we have seen this when $\mathbf{C}$ and sg. $X_{0}$ are smooth from direct computation with $\eta_{*}$ and, in general, from the contact structure on $\mathrm{PT}^{*}(S)$.

\section{Applications.}

EXAMPLE (i). Consider the universal projective hypersurface of degree $d$ in $\mathbb{P}^{n}$ :

$$
\begin{aligned}
\mathbf{X} & \subseteq \mathbb{P}(n, d) \times \mathbb{P}^{n} \\
\downarrow & \\
\mathbb{P}(n, d) . &
\end{aligned}
$$

If $X \subseteq \mathbb{P}^{n}$ is a hypersurface of degree $d$, then $\mathbf{T}_{X}^{1}=\left.\left(N_{X \mid \mathbb{P}^{n}}\right)\right|_{\mathbf{s g} . X}=\left.\mathbf{O}_{\mathbf{p}^{n}}(d)\right|_{\mathbf{s g} . X}$, so the Gauss map $\Phi: \mathbf{C} \rightarrow \mathbb{P} T^{*}(\mathbb{P}(n, d))$ is the universal $d$-uple Veronese map, hence an embedding. Consequently in this example, $\mathbf{C} \cong \hat{D}$; i.e. the critical locus is isomorphic to the Nash blowup of the discriminant locus [S-V4], something which was known to be true for the versal deformation of an isolated hypersurface singularity. Now $\mathbb{P} C_{0}(D)=\bigcup_{\alpha} \phi\left(Z_{\alpha}\right)^{*}$ and since $\phi$ is the Veronese map, of degree $d>1, \phi\left(Z_{\alpha}\right)$ contains no lines, hence the union is precisely the decomposition of $\mathrm{P} C_{0}(D)$ into distinct irreducible components.

Here is a generalization of the family of all projective hypersurfaces.

EXAMPLE (7.1). Linear system on a nonsingular variety.

Let $V$ be a nonsingular variety, $\mathbf{L}$ a line bundle on $V$, and $E \subseteq H^{0}(V, \mathbf{L})$ a vector subspace. The resulting linear system on $V$ is the family of hypersurfaces with base space $S=\mathbb{P}(E)$ and total space $\mathbf{X}=\{(x,[\sigma]) \in V \times \mathbb{P}(E) \mid \sigma(x)=0\}$, $\pi(x,[\sigma])=[\sigma]$. Given $[\sigma] \in \mathbb{P}(E)$, and letting $X$ denote the divisor $X_{[\sigma]}=\pi^{-1}([\sigma])$ of $V$ defined by $\sigma,\left.\left.\mathbf{T}_{X}^{1} \cong \mathbf{N}_{X / V}\right|_{\text {sg. } X} \cong \mathbf{L}\right|_{\text {sg. } X}, \mathbb{P} T_{[\sigma]}^{*}(\mathbb{P}(E))$ is the hyperplane $H$ of $\mathbb{P}(E)^{*}$ dual to $[\sigma]$, and $\phi$ is the rational map: sg. $X \rightarrow H$ induced by the rational map: $V \rightarrow \mathbb{P}(E)^{*}$ defined by the linear system. If the linear system is base point free, $\mathbf{X}$ is nonsingular and $\phi$ is everywhere defined. If, also, $\mathbf{L}$ is ample then $\phi$ is finite so Theorem (4.1) describes the tangent cones to the discriminant of the linear system.

EXAMPLE (ii). Next consider the universal family of theta divisors over Siegel space

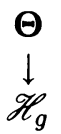

and the fiber $\Theta$ over a point $\Omega$. Let $\operatorname{sg}_{2} \Theta$ denote the double points of $\Theta$.

PROPOSITION (7.2). Let $Z$ be an irreducible component of $\operatorname{sg}_{2} \Theta$ on which $\phi$ is generically finite. Let $V=\overline{\phi(Z)}$ and assume that $V^{*}$ is a hypersurface. Then $V^{*}$ is an irreducible component of $\mathbb{P} C_{\Omega}\left(\mathrm{N}_{0}\right)$. 
ProOF. Recall first, that, since $Z \subseteq \operatorname{sg}_{2} \Theta$, the total space $\Theta$ is nonsingular along $Z$ by the heat equations so $\phi$ is defined on $Z$. Now let $\mathbf{C}_{1}$ be an irreducible component of $\mathbf{C}$ containing $Z$ and $\mu: \mathbf{C}_{1}^{\prime} \rightarrow \mathbf{C}_{1}$ be the blowing-up of $\mathbf{C}_{1}$ along the base locus of $\Phi$ so that $\phi^{\prime}: \mathbf{C}_{1}^{\prime} \rightarrow \mathbb{P} T^{*}\left(\mathscr{H}_{g}\right)$ is everywhere defined. Let $Z^{\prime}=\mu^{-1}(Z)$ and $\bar{Z}^{\prime}$ be its closure in $\mathbf{C}_{1}^{\prime}$. Then $\phi^{\prime}$ is generically finite on $\bar{Z}^{\prime}$ since $\phi$ was assumed generically finite on $Z$ and on $Z^{\prime}, \mu$ is an isomorphism to $Z$. Let $\widetilde{\mathbf{C}}_{1}^{\prime}$ be the blowup of $\mathbf{C}_{1}^{\prime}$ along $\mu^{-1}$ (sg. $\Theta$ ) and $\mathscr{E}^{\prime}$ be an irreducible component of the exceptional divisor of $\widetilde{\mathbf{C}}_{1}^{\prime}$ which maps onto $\bar{Z}^{\prime}$. (Since $\operatorname{sg}_{2} \Theta$ is open in $\operatorname{sg} \Theta, \bar{Z}$ is an irreducible component of sg. $\Theta$ so $\bar{Z}^{\prime}$ is an irreducible component of $\mu^{-1}$ (sg. $\left.\Theta\right)$.) Then, by the proof of $(4.1), \tilde{\phi}^{\prime}\left(\mathscr{E}^{\prime}\right)$ is the dual correspondence of $\phi^{\prime}\left(\bar{Z}^{\prime}\right)=\overline{\phi(Z)}=V$. To make sure that the image of $\mathscr{E}^{\prime}$ in $\mathbb{P} T_{\Omega}\left(\mathscr{H}_{g}\right)$, which is $V^{*}$, is contained in $\mathbb{P} C_{\Omega}\left(\mathbf{N}_{0}\right)$ note that $\tilde{\mathbf{C}}_{1}^{\prime}$ maps to $\tilde{\mathbf{C}}_{1}$, the blowup of $\mathbf{C}_{1}$ along sg. $\Theta$, and hence to $\tilde{\mathbf{N}}_{0}$ the blow up of $\mathrm{N}_{0}$ at $\Omega$. Finally, since $\mathrm{N}_{0}$ has pure codimension 1 in $\mathscr{H}_{g}$ and $V^{*}$ is assumed to be a hypersurface of $\mathbb{P} T_{\Omega}\left(\mathscr{H}_{g}\right), V^{*}$ is an irreducible component of $\mathbb{P} C_{\Omega}\left(\mathbf{N}_{0}\right)$.

Here is a case which was known, by Andreotti-Mayer [Mu2, p. 87], plus deformations of isolated hypersurface singularities.

COROLlaRY (7.3). Let $H_{\xi}$ be the hyperplane of $\mathbb{P} T_{\Omega}\left(\mathscr{H}_{g}\right)$ defined by the quadric tangent cone to $\Theta$ at an isolated double point $\xi$. Then the isolated double point "disappears" in directions out of $H_{\xi}$ and "persists" in directions in $H_{\xi}$.

PROOF. $H_{\xi}=\phi(\{\xi\})^{*}$.

COROllaRY (7.4). For generic $\Omega(C) \in \mathbf{J}_{g}, g \geq 5,{\overline{\phi\left(\operatorname{sg}_{2} \Theta(C)\right)}}^{*}$ is an irreducible component of $\mathbb{P} C_{\Omega(C)}\left(\mathbf{N}_{0}\right)$. ( $\mathbf{J}_{g} \subseteq \mathscr{H}_{g}$ is the Jacobi locus.)

Proof. For $C$ generic, $Z=\operatorname{sg}_{2} \Theta(C)$ is irreducible [F-L, Corollary 2.4] and $\phi$ has degree 2 on $Z$ so it remains to check that $V^{*}$ is a hypersurface, where $V=\overline{\phi\left(\operatorname{sg}_{2} \Theta(C)\right)}$. For this consider a 1-parameter degeneration to a generic trigonal curve $C_{0}$. Now sg. $\Theta\left(C_{0}\right)$ has two irreducible components each birational to $V_{0}$ so, since the abelian variety $J\left(C_{0}\right)$ contains no rational curves, $V_{0}$ is not covered by lines. It follows that $V_{t}$ is not covered by lines for general $t$, thus $V_{t}^{*}$ must be a hypersurface.

The situation simplifies for the generic curve of genus $g \leq 8$ since then all the singularities of $\Theta(C)$ are double points. We will treat the genus 5 case.

COROllary (7.5). For generic $\Omega(C) \in \mathbf{J}_{5}$,

$$
\left.\mathbb{P} C_{\mathbf{J}_{5}}\left(\mathbf{N}_{0}\right)\right|_{\Omega(C)}=\Gamma(C)^{*} \text { as schemes, }
$$

where $\Gamma(C)$ is the quintic discriminant curve in the net $\Pi$ of quadrics containing the canonical curve $C \subset \mathbb{P}^{4}$ and $\Gamma(C)^{*}$ is the dual plane curve.

ProOF. By (7.4), $\phi(\operatorname{sg} . \Theta(C))^{*}$ is an irreducible component of $\mathbb{P} C_{\Omega(C)}\left(\mathbf{N}_{0}\right)$. To show it is the only component we need to check that $\mathbf{C}$ is nonsingular along sg. $\Theta(C)$ and sg. $\Theta(C)$ is nonsingular. These are both proved in [S-V2, Theorem (1.11) and proof, together with Proposition (0.21) (1a) and proof]. Now recall that $\Gamma(C)$, after inclusion of $\Pi=\mathbb{P} N_{\Omega(C)}^{*}\left(\mathbf{J}_{g} / \mathscr{H}_{g}\right)$ in $\mathbb{P} T_{\Omega(C)}^{*}\left(\mathscr{H}_{g}\right)$ is $\phi(\operatorname{sg} \cdot \Theta(C))$. Then the statement about the normal cone follows as sets.

To get the result as schemes, we only need to check that $\left.\mathbf{P} C_{\mathbf{J}_{5}}\left(\mathbf{N}_{0}\right)\right|_{\Omega(C)}$ is reduced. Since sg. $\Theta(C)$ is nonsingular, we may use the multiplicity formula from 
either $\S 5$ or $\S 6$. For example, from (5.11), nonsingularity of $\mathbf{C}$ and sg. $\Theta(C)$ implies $r_{\alpha}=1$, so

$$
\frac{\operatorname{mult}_{0}\left(\mathbf{N}_{0}\right)}{\operatorname{deg}\left(\Gamma(C)^{*}\right)}=\frac{\operatorname{deg}(\phi)}{\operatorname{deg}(\mathbf{C} / D)}
$$

i.e.,

$$
\frac{\operatorname{deg}(\mathbb{P} C)}{\operatorname{deg}\left((\mathbb{P} C)_{\text {red. }}\right)}=\frac{\operatorname{deg}(\phi)}{\operatorname{deg}(\mathbf{C} / D)},
$$

an integer which we claim equals one. It suffices to check $\operatorname{deg}(\mathbf{C} / D) \geq \operatorname{deg}(\phi)$. (Note, since sg. $\Theta(C)$ is connected and $\mathbf{C}$ is nonsingular along it, that $\mathbf{N}_{0}$ has only one component containing $\mathbf{J}_{g}[\mathbf{S}-\mathbf{V} 2]$, so $\operatorname{deg}(\mathbf{C} / D)$ refers to the local degree near $\Omega(C)$.) Since we know the map $\phi: \operatorname{sg} . \Theta(C) \rightarrow \Gamma(C)$, we have from $[\mathbf{A}-\mathbf{M}]$

$$
p \mapsto Q_{p}
$$

that $\operatorname{deg}(\phi)=2$. To see that $\operatorname{deg}(\mathbf{C} / D) \geq 2$, just note that a general jacobian of genus five has an infinite number of singular points hence some which are not points of order two in the group law. Hence the theta divisor over a general point of $\mathbf{N}_{0}$, near $\Omega(C)$, has some singularities which are not fixed by the involution, hence has at least two singular points. Q.E.D.

\section{BIBLIOGRAPHY}

[A-M] A. Andreotti and A. Mayer, On period relations for abelian integrals on algebraic curves, Ann. Scuola Norm. Sup. Pisa 21 (1967), 189-238.

[A1] V. I. Arnold, Mathematical methods of classical mechanics, Graduate Texts in Math., vol. 60, Springer-Verlag, New York, 1978.

[A2] __, Singularities of ray systems, Proc. Internat. Congr. Math. (August 16-24, 1983, Warszawa, vol. 1, PWN, Warsaw, 1984, pp. 27-49.)

[B] A. Beauville, Les singularités du diviseur $\Theta$ de la jacobienne intermediaire de l'hypersurface cubique dans $\mathbf{P}^{4}$, Lecture Notes in Math., vol. 947, Springer-Verlag, New York, 1982, pp. 190-208.

[C-G] C. H. Clemens and P. Griffiths, The intermediate Jacobian of the cubic threefold, Ann. of Math. (2) 95 (1972), 281-356.

[D-S] R. Donagi and R. Smith, The structure of the Prym map, Acta Math. 146 (1981), 25-102.

[D] A. Douady, Flatness and privilege, Enseign. Math. 14 (1968), 47-74.

[Fr] R. Friedman, Global smoothings of varieties with normal crossings, Ann. of Math. (2) 118 (1983), 75-114.

[Fu] W. Fulton, Intersection theory, Springer-Verlag, New York, 1984.

[F-L] W. Fulton and R. Lazarsfeld, On the connectedness of degeneracy loci and special divisors, Acta Math. 146 (1981), 271-283.

[Gra] H. Grauert, Über Modifikationen und Exzeptionelle analytische Mengen, Math. Ann. 146 (1962), 331-368.

[Gre] M. Green, Quadrics of rank four in the ideal of the canonical curve, Invent. Math. 75 (1984), 84-104.

[G-H] P. Griffiths and J. Harris, Principles of algebraic geometry, Wiley, New York, 1978.

[Ha1] R. Hartshorne, Ample subvarieties of algebraic varieties, Lecture Notes in Math., vol. 156, Springer-Verlag, New York, 1970.

[Ha2] _ Algebraic geometry, Springer-Verlag, New York, 1977.

[Hi] H. Hironaka, Resolution of singularities of an algebraic variety over a field of characteristic zero, Ann. of Math. (2) 79 (1964), 109-326.

[H-R] H. Hironaka and H. Rossi, On the equivalence of imbeddings of exceptional complex spaces, Math. Ann. 156 (1964), 313-333.

[I] B. Iverson, Critical points of an algebraic function, Invent. Math. 12 (1971), 210-224. 
[Ke1] G. Kempf, On the geometry of a theorem of Riemann, Ann. of Math. (2) 98 (1973), 178-185.

[Ke2] — Deformations of symmetric products, Riemann Surfaces and Related Topics (Proc. 1978 Stony Brook Conf.), Ann. Math. Stud. 97, Princeton Univ. Press, 1981, pp. 319-341.

[K11] S. Kleiman, About the conormal scheme, Lecture Notes in Math., vol. 1092, Springer-Verlag, New York, 1984, pp. 161-197.

[K12] _ , Tangency and duality, Proc. 1984 Vancouver Conf. in Algebraic Geometry, Amer. Math. Soc., Providence, R. I., 1986, pp. 163-225.

[L-T] Lê, D. T. and B. Teissier, Limites d'espaces tangents en géométrie analytique, preprint, 1986.

[L] E. Looijenga, Isolated singular points on complete intersections, London Math. Soc. Lecture Note Series 77, Cambridge Univ. Press, 1984.

[M-M] A. Mattuck and A. Mayer, The Riemann-Roch theorem for algebraic curves, Ann. Scuola Norm. Sup. Pisa 17 (1963), 223-237.

[Mi] J. Milnor, Singular points of complex hypersurfaces, Ann. Math. Stud. 61, Princeton Univ. Press, 1968.

[Mu1] D. Mumford, Introduction to algebraic geometry, Lecture Notes, Harvard Univ., 1967.

[Mu2] _ C Curves and their Jacobians, Univ. of Michigan Press, Ann Arbor, 1975.

[Mu3] _ Algebraic geometry. I. Complex projective varieties, Springer-Verlag, New York, 1976.

[Mu4] Some footnotes to the work of C. P. Ramanujan, C. P. Ramanujan--a tribute, Tata Inst. Fund. Res. Studies in Math 8, Springer, Berlin, 1978, pp. 247-262. MR 80m: 14026.

[S] C. Sabbbah, Quelques remarques sur la géométrie des espaces conormaux, Systèmes Différentiels et Singularités, Astérisque 130 (1985), 161-192.

[Schl1] M. Schlessinger, Infinitesimal deformations of singularities, Thesis, Harvard Univ., 1964.

[Schl2] __, On rigid singularities, Complex Analysis, 1972, Rice Univ. Stud., vol. 59, No. 1, 1973, pp. 147-162.

[Schu] H. W. Schuster, Deformationen analytischer Algebren, Invent. Math. 6 (1968), 262-274.

[S-V1] R. Smith and R. Varley, On the geometry of $\mathbf{N}_{0}$, Rend. Sem. Mat. Univ. Politech. Torino 42 (1984), $29-37$.

[S-V2] _ Components of the locus of singular theta divisors of genus 5, Algebraic Geometry (Sitges 1983), Lecture Notes in Math., vol. 1124, Springer-Verlag, pp. 338-416.

[SV-3] , The tangent cone to the discriminant, Proc. 1984 Vancouver Conf. in Algebraic Geometry, Amer. Math. Soc., Providence, R. I., 1986, pp. 443-460.

[S-V4] _ Gauss maps and first order deformations of singular hypersurfaces, Bol. Soc. Mat. Méxicana (to appear).

[S-V5] _ Deformations of theta divisors and the rank four quadrics problem, in preparation.

[T1] B. Teissier, Deformations à type topologique constant. I, II, Sem. Douady-Verdier, 1971-72, Astérisque 16 (1974), 215 249.

[T2] _ Cycles evanescents, sections planes et conditions de Whitney, Singularités à Cargèse, 1972, Asterisque 7-8 (1973), 285-362.

[T3] _ The hunting of invariants in the geometry of discriminants, Real and Complex Singularities (Oslo 1976) (P. Holm, ed.), Stijhoff and Noordhoff, 1977, pp. 565-678.

[T4] _ Sur la classification des singularités des espaces analytiques complexes, Proc. Internat. Congr. Math. (August 16-24, 1983, Warszawa), vol. 1, PWN, Warsaw, 1984, pp. 763-781.

[Wei] A. Weinstein, Lectures on symplectic manifolds, CBMS Regional Conf. Ser. in Math., no. 29, Amer. Math. Soc., Providence, R. I., 1977.

[Wel] G. Welters, Polarized abelian varieties and the heat equations, Compositio Math. 49 (1983), 173-194.

[Wh] H. Whitney, Complex analytic varieties, Addison-Wesley, Reading, Mass., 1972.

Departient of Mathematics, University of Georgia, Athens, Georgia 30602 